\title{
Genome-wide analysis of growth-regulating factors (GRFs) in Triticum aestivum
}

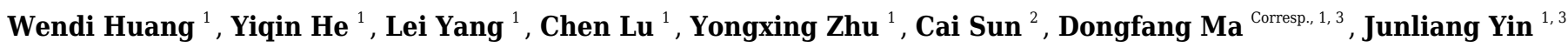 \\ 1 Engineering Research Center of Ecology and Agricultural Use of Wetland, Ministry of Education/Hubei Collaborative Innocation Center for Grain \\ Industry/College of Agriculture, Yangtze University, Jingzhou, Hubei, China \\ 2 Plant Protection and Fruiter Technical Extension Station, Wanzhou District, Chongqing, China \\ 3 Ministry of Agriculture Key Laboratory of Integrated Pest Management in Crops in Central China, Institute of Plant Protection and Soil Science, Hubei \\ Academy of Agricultural Sciences, Wuhan, Hubei, China \\ Corresponding Author: Dongfang Ma \\ Email address: madf@yangtzeu.edu.cn
}

The Growth-Regulating Factor (GRF) family encodes a type of plant-specific transcription factor (TF). GRF members play vital roles in plant development and stress response. Although GRF family genes have been investigated in a variety of plants, they remain largely unstudied in bread wheat (Triticum aestivum L.). The present study was conducted to comprehensively identify and characterize the $T$. aestivum GRF (TaGRF) gene family members. We identified 30 TaGRF genes, which were divided into four groups based on phylogenetic relationship. TaGRF members within the same subgroup shared similar motif composition and gene structure. Synteny analysis suggested that duplication was the dominant reason for family member expansion. Expression pattern profiling showed that most TaGRF genes were highly expressed in growing tissues, including shoot tip meristems, stigmas and ovaries, suggesting their key roles in wheat growth and development. Further qRT-PCR analysis revealed that all 14 tested TaGRFs were significantly differentially expressed in responding to drought or salt stresses, implying their additional involvement in stress tolerance of wheat. Our research lays a foundation for functional determination of TaGRFs, and will help to promote further scrutiny of their regulatory network in wheat development and stress response. 
1 Genome-wide analysis of growth-regulating factors (GRFs) in Triticum aestivum

2 Wendi Huang 1, Yiqin He ${ }^{1}$, Lei Yang ${ }^{1}$, Chen Lu ${ }^{1}$, Yongxing Zhu ${ }^{1}$, Cai Sun ${ }^{3}$, Dongfang Ma ${ }^{1,2 *}$,

3 Junliang Yin ${ }^{1,2}$

$4{ }^{1}$ Engineering Research Center of Ecology and Agricultural Use of Wetland, Ministry of

5 Education/Hubei Collaborative Innocation Center for Grain Industry/College of Agriculture,

6 Yangtze University, Jingzhou 434025, Hubei, P. R. China

$7 \quad{ }^{2}$ Ministry of Agriculture Key Laboratory of Integrated Pest Management in Crops in Central

8 China, Institute of Plant Protection and Soil Science, Hubei Academy of Agricultural Sciences,

9 Wuhan 430064, China

10 3Plant Protection and Fruiter Technical Extension Station, Wanzhou District, Chongqing, China

$11 *$ Corresponding author:

12 Dongfang Ma, E-mail: madf@yangtzeu.edu.cn, College of Agriculture, Yangtze University, 13 Jingzhou 434025, Hubei, P. R. China 


\section{Abstract}

The Growth-Regulating Factor (GRF) family encodes a type of plant-specific transcription factor (TF). GRF members play vital roles in plant development and stress response. Although GRF family genes have been investigated in a variety of plants, they remain largely unstudied in bread wheat (Triticum aestivum L.). The present study was conducted to comprehensively identify and characterize the $T$. aestivum GRF (TaGRF) gene family members. We identified 30 TaGRF genes, which were divided into four groups based on phylogenetic relationship. TaGRF members within the same subgroup shared similar motif composition and gene structure. Synteny analysis suggested that duplication was the dominant reason for family member expansion. Expression pattern profiling showed that most $T a G R F$ genes were highly expressed in growing tissues, including shoot tip meristems, stigmas and ovaries, suggesting their key roles in wheat growth and development. Further qRT-PCR analysis revealed that all 14 tested TaGRFs were significantly differentially expressed in responding to drought or salt stresses, implying their additional involvement in stress tolerance of wheat. Our research lays a foundation for functional determination of TaGRFs, and will help to promote further scrutiny of their regulatory network in wheat development and stress response.

Keywords: GRF; gene expression; abiotic stress; growing development; qRT-PCR

\section{Introduction}

Growth-Regulating Factors (GRFs) are plant-specific transcription factors that play important roles in regulating plant growth and abiotic stress response (Kim et al., 2012; Baucher et al., 2013). The first GRF gene $O s G R F 1$ was identified from rice, where it was shown to play an essential role in regulating gibberellic acid (GA)-induced stem elongation (van der Knaap, Kim \& Kende, 2000). In recent years, with the development of reference genomes, many GRF genes have been identified 
and characterized from plant species at genome-wide levels (Omidbakhshfard et al., 2015). Protein sequence analysis has determined that there are two conserved domains, QLQ (Gln, Leu, Gln) and WRC (Trp, Arg, Cys), in the N-terminal region of the GRF protein (Kim, Choi \& Kende, 2003). The QLQ domain serves as a protein-protein interaction feature which can interact with the GRFinteracting factor (GIF) (Kim \& Kende, 2004). The WRC domain is mainly involved in DNA binding and consists of a functional nuclear localization signal and a DNA binding motif (zinc finger structure) (Choi, Kim \& Kende, 2004). Unlike the conserved amino acid residues in the Nterminal region, the $\mathrm{C}$-terminal region of GRF is variable, with some studies demonstrating that the C-terminal region has trans-activation activity (Choi, Kim \& Kende, 2004; Kim \& Kende, 2004; Liu et al., 2014). In addition, the C-terminal region may contain several low conservative motifs, such as TQL (Thr, Gln, Leu) and FFD (Phe, Phe, Asp) (Zhang et al., 2008).

Currently, the GRF transcription factors have been reported in Arabidopsis (Kim, Choi \& Kende, 2003), rice (Choi, Kim \& Kende, 2004), maize (Zhang et al., 2008), Chinese cabbage (Wang et al., 2014), soybean (Chen et al., 2019) and tea (Wu, Wang \& Zhuang, 2017). In these plants, GRF genes are strongly expressed in tissues involved in active growth and development, such as stem tips, flower buds, and immature leaves, but weakly expressed in mature tissues or organs. They can participate in the early growth and development of plants and play an important regulatory role in the formation of plant tissues or organs, such as leaf development, stem elongation and root growth (Bazin et al., 2013; Kuijt et al., 2014; Wu et al., 2014). GRF genes have been reported as positive regulators of leaf size by promoting and/or maintaining the proliferation activity of leaf primordia cells (Horiguchi, Kim \& Tsukaya, 2005; Kim \& Lee, 2006). For example, overexpression of $A t G R F 1, A t G R F 2$, and $A t G R F 5$ resulted in larger than normal leaves in wild-type (WT) Arabidopsis, while the leaves of grf mutants, such as grf3-1, grf5-1, grf1- 
$611 / g r f 2, \operatorname{grf} 2 / g r f 3$, and $g r f 1 / 2 / 3$, were much smaller than the WT (Debernardi et al., 2014;

62 Horiguchi, Kim \& Tsukaya,, 2005). GRF2 was found to enhance seed oil production in rapeseed

63 (Brassica napus) by regulating cell number and plant photosynthesis (Liu et al., 2012). GRF TFs

64 not only participate in plant growth and development, but also respond to certain abiotic stresses

65 (Kim et al., 2012). In Arabidopsis, while under stress conditions AtGRF7 expression is inhibited

66 to activate osmotic stress-responsive genes (Kim et al., 2012). Functional classification of the

67 AtGRF1 and AtGRF3 downstream genes suggests that most target genes are involved in defense

68 responses and disease resistance processes (Liu et al., 2014).

69 Although bread wheat is one of the world's most important food crops, accounting for more than half of total human consumption (Ma et al., 2016; Sun et al., 2018; Yin et al., 2018a), its production is seriously threatened by biotic and abiotic stress factors, including drought, salinity, and extreme temperatures (Yin et al., 2019; Zhu et al., 2015; Zhu et al., 2019a). Although genomewide analyses of GRF transcription factors have been performed to a number of plant species, including Arabidopsis (Kim, Choi \& Kende, 2003), rice (Choi, Kim \& Kende, 2004), maize (Zhang et al., 2008), Chinese cabbage (Wang et al., 2014), soybean (Chen et al., 2019) and tea (Wu, Wang \& Zhuang, 2017), genome-wide identification and characterization have not yet been conducted to common wheat GRF (TaGRF) family members.

In this study, bioinformatics methods were used to systematically analyze the TaGRF TFs, including sequence characteristics, chromosome distribution, phylogenetic relationship, gene structure, and conserved motif and domain prediction. In total, 30 TaGRFs were identified from the wheat genome. On this basis, the gene expression patterns of wheat GRF were analyzed based on RNA-seq data from different wheat tissues. The expression patterns of TaGRFs under drought and salt stresses were also analyzed by qRT-PCR. 
84

85

86

87

88

89

90

91

92

\section{Material and Methods}

\section{Identification of GRF genes in T. aestivum, T. urartu, T. dicoccoides and Ae. tauschii}

Genome-wide data for Triticum aestivum (IWGSC v1.1), Triticum urartu (v1.43), Triticum dicoccum (v1.0.43), and Aegilops tauschii (v4.0.43) were downloaded from Ensembl Plants database (http://plants.ensembl.org/index.html). First, the Hidden Markov Model (HMM) of WRC (PF08879) and QLQ (PF08880) domains were obtained from PFAM (http://pfam.xfam.org/) and used as query sequences for HMMER3.0 (http://hmmer.org/download.html) searching (e-value $\left.\leq 1 \mathrm{e}^{-10}\right)$. Second, download of known GRF protein sequence were used as query sequences, including 9 GRFs from Arabidopsis (Berardini et al., 2015), 14 GRFs from Zea mays (Andorf et al., 2015), and 12 GRFs from Oryza sativa (Ouyang et al., 2007). They were then used as query sequences for BLASTp searching the wheat database. The first uncurated protein sequence list was genereted by e-values lower than $1 \times 10^{-10}$. Next, we combined the results and deleted the redundant sequences. Finally, predicted proteins were considered as GRFs only if they contained

QLQ and WRC conserved domains verified by NCBI CDD (https://www.ncbi.nlm.nih.gov/Structure/cdd/wrpsb.cgi) and SMART (http://smart.embl-heidelberg.de/) (Letunic \& Bork, 2017).

\section{Characterization of TaGRFs Proteins}

ExPASy server10 (https://web.expasy.org/compute_pi/) was used to predict the amino acid length, molecular weight (MW), isoelectric point (pI), stability, and grand average of hydropathicity (GRAVY) for TaGRFs proteins (Li et al., 2018); and subcellular localization prediction was carried out by Plant-mPLoc (http://www.csbio.sjtu.edu.cn/bioinf/plant-multi/) 
105 (Chou \& Shen, 2010).

106 Chromosomal Location and Gene Duplication of TaGRFs

107 The wheat genome GFF3 gene annotation file was from the wheat database IWGSC v1.1 108 (https://wheat-urgi.versailles.inra.fr/Seq-Repository/Assemblies). Gene structure annotations of

109

110

111

112

113

114

115

116

117

118

119

120

121

122

123

124

125

TaGRFs were extracted from the GFF3 file. The start and end location information of the TaGRFs

in the corresponding chromosomes was used to draw the physical map by the software MapInspect

(Fang et al., 2019). The orthologous genes from wheat and its subgenome donor were identified

by the common tool "all against all BLAST search". The cutoff values (e-value $<10^{-10}$, identity $>$ $80 \%$ ) were used to assure the reliability of the orthologues. Then we used Multiple Collinearity

Scan toolkit (MCScanX) to depict their homology relationships (Wang et al., 2012). Synteny diagrams were generated using the R package "circlize". Gene duplication events were divided into tandem duplication events and segmental duplication events. Tandem duplication events were determined by the following evaluation criteria: (1) length of the aligned sequence $>80 \%$ but of each sequence, (2) identity $>80 \%$, (3) threshold $\leq 10^{-10}$, (4) only one duplication can be recognised when genes are tightly linked; and (5) intergenic distance is less than $25 \mathrm{~kb}$. When genes passed the criteria for (1), (2), and (3), but were on a different chromosome, they were deemed to be segmental duplications (Fang et al., 2020; Jiang et al., 2020).

\section{Analysis of TaGRFs gene structures and motifs}

According to the TaGRFs annotation information, GSDS2.0

(http://gsds.cbi.pku.edu.cn/index.php) was used to produce TaGRFs genetic structure (Hu et al., 2017). The MEME v4.9.1 (http://meme-suite.org/) was used to identify conserved TaGRF protein 
126

127

128

129

130

131

132

133

134

135

136

137

138

139

140

141

142

143

144

145

146

motifs (Zheng et al., 2017). The trained parameters were applied as follows: each sequence may contain any number of nonoverlapping occurrences of each motif, up to 20 different motifs, and a motif width range of 6 to 50 amino acids (aa). These motif patterns were drawn using TBtools software (Chen et al., 2018). The annotations of those predicted motifs were analyzed by SMART (http://coot.embl-heidelberg.de/SMART/) (Letunic \& Bork, 2017). Multiple amino acid sequences were aligned using DNAMAN6.0 (Lynnon Biosoft).

\section{Phylogenetic analyses of TaGRFs}

The 99 protein sequences ( 9 AtGRFs, 12 OsGRFs, 14 ZmGRFs, 30 TaGRFs, 6 TuGRFs, 10 AeGRFs, and 18 TdGRFs) were conducted multiple comparisons by using ClustalW2 software (Thompson, Higgins \& Gibson, 1994). Then, the phylogenetic relationships were inferred using the Neighbor-Joining (NJ) method with bootstrap analysis for 1000 repetitions by MEGA7.0 (Kumar, Stecher \& Tamura, 2016). Finally, the midpoint rooted base tree was drawn using Interactive Tree of Life (IToL, v4, http://itol.embl.de) (Letunic \& Bork, 2019).

\section{Cis-acting elements analysis of TaGRFs}

The PlantCARE (http://bioinformatics.psb.ugent.be/webtools/plantcare/html/) was used to predict cis-acting elements in the regions $1500 \mathrm{bp}$ upstream of $30 \mathrm{TaGRFs}$ start codons (Lescot et al., 2002). The predicted results were organized and displayed by the R package "pheatmap" (Jiang et al., 2019; Zhu et al., 2019b).

\section{Gene Ontology annotation in TaGRF family genes}

The functional annotation of GRF sequences and the analysis of annotation data were performed using Blast2GO (http://www.blast2go.com) (Conesa and Götz, 2008). The full-length amino acid 
147 sequence of the TaGRF proteins were uploaded to the original program, drawn and annotated. The 148 program provides the output defining three categories of GO classification namely biological 149 processes, cellular components, and molecular functions.

150

151

152 153 154 155 156 157 158 159 160

\section{Multiple conditional transcriptome analysis of TaGRFs}

The multiple transcriptome data were downloaded from the Wheat Expression Browser (http://www.wheat-expression.com/) (Ramírez-González et al., 2018); and the heat maps of $T a G R F S$ were generated using the R package "pheatmap".

\section{Growth and stress treatment of wheat seedlings}

Seeds of Emai 170 (a hexaploid common wheat cultivars) were sterilized on the surface with $1 \%$ hydrogen peroxide, rinsed thoroughly with distilled water, and germinated in an incubator at $25^{\circ} \mathrm{C}$ for 2 days (He et al., 2020; Ma et al., 2016). According to the reported method, the seedlings were transferred to $1 / 2$ strength Hoagland nutrient solution and cultured in continuous ventilation (Yin et al., 2019; Zhu, Gong \& Yin, 2019c). After five days (when wheat seeding reached the stage of one heart and one leaf), $85.5 \mathrm{mM} \mathrm{NaCl}$ and $82.5 \mathrm{mM}$ mannitol were applied to seedings. Every two days, $2 \mathrm{M} \mathrm{KOH}$ or $0.4 \mathrm{M} \mathrm{H}_{2} \mathrm{SO}_{4}$ was used to adjust the $\mathrm{pH}$ of culture solution to 6.0. During the application, the plants were grown at $16 \mathrm{~h} / 8 \mathrm{~h}$ (day/night) and $25{ }^{\circ} \mathrm{C}$. Leaves and roots were collected at $2 \mathrm{~h}, 4 \mathrm{~h}, 8 \mathrm{~h}, 12 \mathrm{~h}, 24 \mathrm{~h}, 96 \mathrm{~h}$ and $144 \mathrm{~h}$ after treatments. Three biological repeats are included for each treatment. Finally, the samples were immediately frozen with liquid nitrogen and stored at $-80^{\circ} \mathrm{C}$.

\section{RNA isolation and $q R T-P C R$ analysis}

According to the manufacturer's instructions, total RNA of samples were extracted by TRizol 
168

169

170

171

172

173

174

175

176

177

178

179

180

181

182

183

184

185

186

187

188

189

190

reagent (Invitrogen, U.S.A) and cleansing DNA with DNaseI (TaKaRa, U.S.A). The first cDNA was reverse-transcribed from RNA by RevertAid Reverse Transcriptase (Vazyme, China). Genespecific primers were designed using Primer 5.0; and the ADP-ribosylation factor Ta2291 (F: GCTCTCCAACAACATTGCCAAC, R: GCTTCTGCCTGTCACATACGC) was used as an internal reference gene for qRT-PCR analysis (Paolacci et al., 2009). The qRT-PCR reaction system and protocol were carried out as manufacturer's instructions for SYBR ${ }^{\circledR}$ (Vazyme, China). For each sample, settings included three technical replicates. Relative gene expression level was calculated using the $2^{-\Delta \Delta \mathrm{Ct}}$ method (Yin et al., 2018b).

\section{Results}

\section{Identification and Analysis of Wheat GRF Transcription Factor Gene Family Members}

For identification of GRF TF genes in wheat, both BLAST and Hidden Markov Model (HMM) searches were performed. The 35 known GRF proteins (Table S1), including Arabidopsis (9), maize (14), rice (12) as the query sequences to conduct BLASTp against the wheat reference genome IWGSCv1.1. Using the HMM of WRC (PF08879) and QLQ (PF08880) domains were used as the query sequences for HMMER3.0 searching. The candidate proteins were verified by NCBI CDD and SMART Online Tools to determine that the TaGRF contained both WRC and QLQ domains. Finally, a total of 30 TaGRFs were identified from the wheat genome. We named wheat GRF genes (TaGRFs) according to the naming rule of Susanne et al. (2020); the corresponding gene IDs are shown in Table 1. Using the same method, we identified 6, 10, and 18 GRFs from T. urartu, Ae. tauschii and T. dicoccoides, respectively (Table S2). The deduced polypeptides ranged in length from 206 (TaGRF2-2A) to 611 (TaGRF4-4B) amino acids, with the predicted molecular weights ranging between 21.6 to $64.2 \mathrm{kDa}$. Their isoelectric points ranged from 4.72 (TaGRF1-2B) to 10.23 (TaGRF2-2A). Their instability parameters were between 41.46 
191 (TaGRF7-6A) to 66.25 (TaGRF12-7B). Their average hydrophilicity coefficient ranged from 1920.269 (TaGRF2-2A) to 0.882 (TaGRF12-7B) (Table 1). Subcellular localization predictions 193 showed that all GRF proteins except TaGRF1-2A and TaGRF2-2A were localized only in the 194 nucleus, while TaGRF1-2A was located in the chloroplast, cytoplasm and nucleus, and TaGRF2195

2A was located in the chloroplast and nucleus.

According to the phylogenetic relationships (Fig. 1), the 30 TaGRFs could be divided into four sub-categories (Group I to IV). Group I consisted of a single member, TaGRF7-6A. Group II included TaGRF1-2A, TaGRF2-2A, TaGRF1-2B, TaGRF2-2B, TaGRF1-2D, TaGRF2-2D, TaGRF8-6A, TaGRF8-6B, and TaGRF8-6D. Group III consisted of TaGRF4-4A, TaGRF5-4A, TaGRF4-4B, TaGRF5-4D, and TaGRF4-4D. The remaining TaGRF genes were classified in Group IV.

The $T a G R F$ gene structure map showed that all wheat GRF gene members contain 1 to 4 introns, with the majority having 2 to 3 introns (Fig. 1, and Table S3). There were 2 to 5 exons, with most $T a G R F$ having 2 to 4 exons. The exon number of $T a G R F$ genes within same group were relatively consistent.

Conservative motif analysis indicated that TaGRF protein domains are highly conserved among the 30 members. Each member contains only two structural domains: WRC (Motif 1) and QLQ (Motif 2) (Fig. 1). Lengths and the most matching sequences of 20 motifs were shown in Table S4.

In order to further analyze the conservation degree of QLQ and WRC domains in TaGRFs, we performed multiple sequence alignment of these two domains. The results indicate that, as highlighted in Fig. 1, the QLQ and WRC motifs are highly conserved. The N-terminal QLQ motif was conserved with one Leu and two Gln in all the TaGRF proteins. The WRC motif was also 
214 highly conserved with one Trp, Arg, and Cys in each of the TaGRF proteins. A zinc finger motif

215 (CCCH) was also found within the WRC domain in all TaGRF proteins (Fig. 2).

216 Chromosome Localization of Wheat TaGRF Genes

217 Based on the GFF3 genome reference files, the chromosome map of TaGRF genes was 218 generated using MapInspect software (Fig. 3, and Table S3). The three sub-genomes A, B, and D 219 contained 15, 11, and $10 T a G R F s$, respectively. But the TaGRFs are not uniformly distributed 220 among chromosomes (chromosome 2, 9; chromosome 4, 6; chromosome 6, 10; and chromosome 221 7, 5). However, distribution range of genes in different group was diverse. Members of TAGRF group II are only distributed on chromosome 4.

\section{Phylogenetic Analysis of GRF Transcription Factor Family Members in Wheat, Rice, Maize,}

\section{Arabidopsis, T. urartu, Ae. tauschii and T. dicoccoides}

The phylogenetic analysis of wheat (30), rice (12), maize (14), Arabidopsis (9), T. urartu (6), Ae. tauschii (10) and T. dicoccoides (18) GRFs showed that 99 GRFs could be divided into 4 subcategories (Group I to IV) (Fig. 4). Group I contained only AtGRF7 and AtGRF8 of Arabidopsis, TaGRF7-6A of wheat. The Group II included 9 TaGRFs, 3 AtGRFs, 2 ZmGRFs, 3 OsGRFs, 3 AeGRFS, 6 TdGRFs, and 3 TuGRFs. The group III consisted of 5 TaGRFs, 2 AtGRFs, 3 ZmGRFs, 4 OsGRFs, 2 AeGRFs, and 4 TdGRFs. The Group IV included, 15 TaGRFs, 2 AtGRFs, 5 OsGRFs, 9 ZmGRFs, 5 AeGRFs, 8 TdGRFs, and 3 TuGRFs. In addition, we found that in Group II - IV, TaGRF has a closer phylogenetic relationship with TuGRF, AeGRF and TdGRF, followed by OsGRF and ZmGRF. and relatively distant from the AtGRF.

\section{TaGRF Gene Promoter Cis-element Analysis}

Analysis of the cis-elements in the promoter sequence was important for understanding the 
236 regulatory functions of genes. The cis-acting element analysis was performed in Plant-CARE by

237 using upstream sequences $(1.5 \mathrm{~kb})$ of $T a G R F$ genes extracted from the wheat genome. The detailed

238 information including function and location were displayed in Table S5. The results showed that

239 all 30 TaGRF genes contained several TATA boxes and CAAT boxes, indicating that TaGRF

240 genes can be normally transcribed. When focusing on the cis-acting elements associated with

241 wheat growth and development, hormonal and stress responses, it can be seen in Fig. 5 the $T a G R F$

242 gene promoter contains a large number of cis-elements, with the largest number found in TaGRF9-

$2436 D$ having 19 cis-elements, and TaGRF3-2A with the least, containing only 8 cis-elements. There

244 were several different light-related elements in these cis-elements, such as AE-box, Box 4, I-box,

245 C-box, Sp1, circadian, CAG-motif, 3-AF1 binding site, LAMP-element, TCT-motif, GATT-motif,

246 ATCT-motif, and Gap-box. This suggests that the GRF gene family may play a role in light 247 response.

248 In addition, a large number of responsive hormones and stress-related cis-elements were found 249 in the promoter region of the TaGRFs, including auxin (11 TGA-elements), gibberellin (8 GARE250 motifs and 6 P-boxs), jasmonic acid methyl ester (65 TGACG-motifs), abscisic acid (72 ABREs) 251 and other hormone response components, as well as anaerobic induction (25 AREs), drought (19

252 MBSs) and low temperature (3 LTRs) and other stress response cis- elements. This suggests a 253 potential role for the wheat GRF family in wheat growth and development and in a variety of 254 hormones and stress.

255 Gene Ontology annotation in TaGRF family genes

256 The GO item analysis was performed using Blast2Go and the results indicated the putative 257 participation of 30 TaGRF proteins in diverse biological processes (Fig. 6, and Table S6). Total 258 ten different GO items of biological processes were defined. Majority of the TaGRFs were 
259

260

261

262

263

264

265

266

267

268

269

270

271

272

273

274

275

276

277

278

279

predicted to function in 'regulation of transcription, DNA-templated (GO: 0006355)' (76.67\%), followed by 'response to deep water (GO: 0030912)' (20\%) and 'response to gibberellin (GO: 0009739)' (20\%). Molecular function prediction showed that about $76.67 \%$ of the TaGRFs were evidenced to participation of 'ATP binding (GO: 0005524)' and 'hydrolase activity, acting on acid anhydrides, in phosphorus-containing anhydrides (GO: 0016818)'. Cellular localization prediction indicated that the majority of TaGRF proteins (80\%) were localized in the nucleus (Fig. 6).

\section{Homologous Gene Pairs and Synteny Analysis}

Gramineae evolved 50-70 million years ago, and the Pooideae subfamily, which includes barley and wheat, evolved about 20 million years ago (Inda et al., 2008; Peng, Sun \& Nevo, 2011). Obviously, common wheat has a complicated evolutionary history, and its ancestors' origin is affected by many factors, but research shows that wheat has two major polyploid evolutionary events (Ling et al., 2013). Homology reflects the phylogeny of a species, so it can be used to transfer annotations for one known gene to another newly sequenced genome. In order to further infer the evolutionary origin and homology of the wheat GRF family, Sixty-four GRFs were identified from T. aestivum (30 TaGRFs), T. urartu (6 TuGRFs), T. dicoccoides (18 TdGRFs) and Ae. tauschii (10 AeGRFs) using a computer-based method (Fig. 7 (A), and Table S7). There were no paralogous gene pairs in Ae. tauschii and T. urartu, and 21 and 5 paralogous gene pairs in $T$. aestivum and T. dicoccoides, respectively. Among them, 18 orthologous gene pairs were identified from T. aestivum and Ae. tauschii, 5 orthologous gene pairs were identified from T. dicoccoides and Ae. tauschii, 5 orthologous gene pairs were identified from T. urartu and Ae. tauschii, 20 orthologous gene pairs were identified from T. aestivum and T. dicoccoides, 12 orthologous gene 
pairs were identified from T. aestivum and T. urartu, and 3 orthologous gene pairs were identified

281

282

283

284

285

286

287

288

289

290

291

292

293

294

295

296

297

298

299

300

301

302

from T. urartu and T. dicoccoides. Given phylogenetic analyses and homology results of four wheat species, it was speculated that 8 TaGRFs (TaGRF2-2B, TaGRF3-2B, TaGRF4-4A, TaGRF4-4B, TaGRF6-4A, TaGRF8-6B, TaGRF9-6A and TaGRF9-6B) originated from $T$. dicoccoides, 9 TaGRFs (TaGRF2-2D, TaGRF3-2D, TaGRF4-4D, TaGRF5-4D, TaGRF8-6D, TaGRF9-6D, TaGRF10-6D, TaGRF11-7D and TaGRF12-7D) from Ae. tauschii, and 5 TaGRFs

(TaGRF3-2A, TaGRF8-6A, TaGRF8-6B, TaGRF10-6A and TaGRF12-7A) from T. urartu.

\section{Gene Expression Pattern Analyses of TaGRFs}

For multigene families, analysis of gene expression patterns often provides useful clues for determining gene function. Transcriptome data from growth and abiotic stresses were downloaded from Wheat Expression Browser to examine their expression patterns. The results showed that 28 TaGRF genes (except TaGRF2-2A and TaGRF7-6A) were expressed in different tissues or under different stress treatments (Fig. 8, and Table S8). TaGRF1-2A and TaGRF1-2D were highly expressed in various tissues. TaGRF5-4A and TaGRF5-4D had the highest expression in shoot tip meristem. Most of the genes were expressed in shoot tip meristems more significantly than other tissues. About half of the TaGRF genes were expressed under $\mathrm{NaCl}$ treatment, and TaGRF4-4A, TaGRF4-4B and TaGRF4-4D were significantly expressed under $\mathrm{NaCl}$ treatment. It was speculated that TaGRF family members may play important roles in the development of wheat shoot tip meristems, and TaGRF4 may play an important role in wheat salt tolerance.

\section{Quantitative-Real Time PCR Analysis}

To further understand the potential role of the TaGRF genes in abiotic stresses $(\mathrm{NaCl}$ and mannitol), qRT-PCR was used to analyze the expression pattern of TaGRFs. Based on transcriptome analysis, we selected $14 T a G R F s$ for qRT-PCR. In the two treatments of this study, 
303 the expression of all $14 T a G R F s$ differed from the control, although their degree of difference was

304 often substantial (Fig. 9).

305 After treatment with $\mathrm{NaCl}$, the expression of TaGRF4-4A, TaGRF4-4D, TaGRF5-4A, 306 TaGRF10-6A, TaGRF 10-6B and TaGRF10-6D were higher in treated leaves than in the control 96 $307 \mathrm{~h}$ after treatment. Expression of the genes TaGRF1-2B, TaGRF1-2D, TaGRF3-2D, and TaGRF9$3086 D$ were lower in the leaves $96 \mathrm{~h}$ after treatment than in the control group. However, in the roots, 309 the expression of TaGRF1-2B and TaGRF3-2D were much higher than in the control $96 \mathrm{~h}$ after 310 treatment, while the expression of TaGRF4-4A, TaGRF4-4D, TaGRF10-6B, and TaGRF10-6D 311 were much lower than in the control $96 \mathrm{~h}$ after treatment. Among these, the expression trends of TaGRF1-2B, TaGRF3-2D, TaGRF4-4A, TaGRF4-4D, TaGRF10-6B, and TaGRF10-6D were completely reversed in the roots compared to the leaves $96 \mathrm{~h}$ after treatment.

After treatment with mannitol, TaGRF1-2B, TaGRF6-4A, TaGRF10-6A and TaGRF9-6D were expressed higher in the roots than in the control group $24 \mathrm{~h}$ and $96 \mathrm{~h}$ after treatment. However, in the leaves, only TaGRF6-4A, TaGRF10-6A and TaGRF9-6D was expressed higher than the control group at $24 \mathrm{~h}$ and $96 \mathrm{~h}$ after treatment. The expression level of TaGRF1-2B did not change compared with the control group. TaGRF1-2B and TaGRF3-2B were significantly down-regulated in leaves at 2 and 4 hours after treatment.

\section{Discussion}

With the in-depth development of plant genomics research, especially the rapid development of sequencing technology, the entire genome sequencing of many plant species has been completed, providing favorable conditions for the identification of plant gene families. GRF transcription factors are plant-specific transcription factors. In recent years, GRF transcription factor members 
326 these studies indicate that GRF genes are mainly expressed in plant meristems and play important

327 roles in plant growth and development. In general, the number of GRF transcription factor

328 members in terrestrial plants is between 8 to 20, but, typically, fewer are found in lower plant taxa

329 such as mosses and algae (Omidbakhshfard et al., 2015). For example, 9 AtGRFs occur in

330 Arabidopsis (Kim, Choi \& Kende, 2003), 12 OsGRFs in rice (Choi, Kim \& Kende, 2004), 14

331 ZmGRFs in maize (Zhang et al., 2008), 17 BrGRFs in Chinese cabbage (Wang et al., 2014), 9

332 CsGRFs in sweet orange (Citrus sinensis L. Osbeck) (Liu et al., 2016), 18 PeGRFs in bamboo (He

333 et al., 2018), while the moss (Physcomitrella patens) has only 2 GRFs (Omidbakhshfard et al.,

334 2015). Based on wheat genomic data, the present study identified 30 wheat GRF transcription

335 factors (TaGRF). Although the wheat genome is considerably larger than the Arabidopsis genome

336 (16 GB vs $125 \mathrm{MB}$ ), the number of GRF genes in wheat is only three times that of Arabidopsis

337 (30:9), indicating that there is a large amount of gene loss during genome replication in wheat.

338 According to phylogenetic analysis, the 30 GRF transcription factors in wheat could be divided

339 into four groups. Studies have shown that GRF transcription factors of rice and maize can be

divided into three groups, five groups in Arabidopsis, and six in rapeseed, indicating that GRF

transcription factors in monocotyledons different from dicotyledons in evolution patterns and

characteristics. In this study, phylogenetic analysis of GRFs of wheat, Arabidopsis, rice, maize, $T$.

urartu, T. dicoccoides and Ae. tauschii were carried out compared. It was shown that most TaGRFs

preferentially clustered with GRF in T. urartu, T. dicoccoides and Ae. tauschii, followed by rice and maize. The results showed that the GRFs in wheat were closely related to those in T. urartu,

to III, implying that the variability in the number of GRF genes in the different groups may be the 
349 structure is important for understanding evolutionary and functional relationships (Hu \& Liu,

350 2011). In addition, gain or loss events in exons or introns provide structural and functional

351 differentiation (Xu et al., 2012). With regard to corresponding gene structures within each group,

352 most $T a G R F$ genes shared a similar gene structure, having two to four introns/exons, which is in

353 accordance with Arabidopsis and rice (Choi et al. 2004; Kim et al. 2003). 22 of the TaGRF genes

354 contained three or four exons and 29 of the TaGRF genes contained two or three introns. This

355 indicated that, the structural evolution of the TaGRF gene is conservative to some extent.

356 Gene replication events are the main drivers of genome and genetic system evolution (Moore \&

357 Purugganan, 2003). Wheat has a complex evolutionary history with two major polyploid events

358 (Ling et al., 2013). About 50-70 million years ago, before the genetic grouping of herbs, the first

359 genome duplication directly produced an ancient doubling event. The second time was that the

360

361

362

363

364

365

366

367

368

369

traceability of common wheat originated from the forming process of the tetraploid wheat $(T$.

dicoccoides, A and B sub-genome) which hybridized by sub-genome progenitor T. urartu and Aegilops speltoides (B sub-genome) 300,000 years ago approximately. Again, about 8000 years ago, the tetraploid wheat was hybridized with Ae.tauschii (D sub-genome) and formed hexaploid wheat (T. aestivum, A, B, and D sub-genome) naturally. We found that some genes were deleted during polyploidization by comparing GRF genes of T. aestivum, T. urartu, Ae. tauschii and T. dicoccoides.

Studies have shown that the expression level of the $G R F$ gene is significantly higher in developing tissues than in mature tissues (Kim, Choi \& Kende, 2003; Kim \& Kende, 2004; Choi, Kim \& Kende, 2004). For example, the $G R F$ genes in rice were found to be strongly expressed in 
370

371

372

373

374

375

376

377

378

379

380

381

382

383

384

385

386

387

388

389

390

buds, immature leaves, and flower buds, and participates in plant growth and development by regulating cell proliferation in actively growing tissues (Choi, Kim \& Kende, 2004). The expression profile of $T a G R F$ genes analyzed by wheat tissue transcriptome data, showed that most of the TaGRF gene is highly expressed in wheat shoot tip meristems, and weakly expressed in other relatively mature tissues, which was similar to the previous conclusions. OsGRF6 participates in regulating the growth and development of rice infloreses(Gao et al., 2015), and the three genes with the highest homology level, TaGRF4-4A, TaGRF4-4B and TaGRF4-4D, have high expression in stigma and ovary, indicating that their function may be related to the growth and development of stigma and ovary. In addition, cis-acting elements related to the regulation of meristem expression, such as cat-box and CCGTCC motif, were found in the promoter region of $T a G R F$ genes, indicating that the $T a G R F$ genes play important roles in wheat growth tissues, especially in stem tip meristems.

Plants have evolved a series of signal pathways and defense systems to resist stresses. In previous researches, the activation of genes responsing stresses enhanced the plant's tolerance (Heidel et al., 2004; Sakuma et al., 2006). Over-expression of AtGRF7 in Arabidopsis under stress conditions increased resistance to osmotic and drought stress (Kim et al., 2012). It has been reported that GRF transcription factors acted as key roles in plant growth by coordinating stress responses and defense signals (Casadevall et al., 2013; Liu et al., 2014). For example, Arabidopsis growth regulators 1 and 3 (AtGRF1 and $A t G R F 3$ ) played significant roles in the regulation of plant growth, defense signals, and stress responses (Casati, 2013; Hewezi et al., 2012). In our study, the cis-elements of 12 TaGRFs (TaGRF1-2A, TaGRF1-2B, TaGRF1-2D, TaGRF3-2D, TaGRF4-4A, 
391

392

393

394

395

396

397

398

399

400

401

402

403

404

405

406

407

408

409

410

411

412

TaGRF5-4A, TaGRF6-4A, TaGRF9-6A, TaGRF9-6B, TaGRF9-6D, TaGRF12-7A, and TaGRF12-

7D) contained 1 to 2 copies of MBS (the MYB binding site is involved in drought-inducing). The qRT-PCR results showed that under $\mathrm{NaCl}$ stress and mannitol simulated drought stress, $14 \mathrm{TaGRF}$ genes we tested responded to external abiotic stresses, either positively or negatively. Among them, 9 genes (TaGRF1-2B, TaGRF3-2B, TaGRF3-2D, TaGRF4-4A, TaGRF4-4D, TaGRF6-4A, TaGRF9-6D, TaGRF10-6A, TaGRF10-6B) were significantly expressed in treatment with $\mathrm{NaCl}$ and mannitol. These genes may play an active role in wheat's response to $\mathrm{NaCl}$ stress and drought stress. According to transcriptomic data and qRT-PCR results, TaGRF1-2D, TaGRF4-4A and TaGRF4-4D were all up-regulated in salt stress, indicating that they may play a certain role in wheat response to salt stress. But more experimental evidence is needed to understand how they work in wheat in response to salt stress.

\section{Conclusions}

This study provides a reference point for subsequent studies involving functions of the $T a G R F$ gene family. TaGRF gene family has extensive expression profiles which span multiple developmental stages and stresses, implying their crucial roles in various physiological functions and abiotic stresses. In summary, our findings provide new clues that will be useful for improving stress tolerance of wheat.

\section{Acknowledgements}

D. F. Ma and J. L. Yin conceived the study. W. D. Huang and Y. X. Zhu designed the experiments and wrote the manuscript. L. Yang and C. Lu performed the plant growth and sampling. Y. Q. He and C. Sun carried out the qRT-PCR analysis. All authors reviewed and approved the final manuscript. 


\section{Funding}

414 This study was funded by the Key Projcet of Hubei Provience Departmen of Education (grant 415 number D20191305).

\section{References}

417 Andorf, CM, Cannon, EK, Portwood, JL, II, Gardiner, JM, Harper, LC, Schaeffer, ML, Braun, 
440

441

442

443

444

445

446

447

448

449

450

451

452

453

454

455

456

457

458

459

460

461

462

463

464

465

466

plant-specific putative transcription activators in rice (Oryza sativa L.). Plant and Cell Physiology 45: 897-904.

Chen, F, Yang, Y, Luo, X, Zhou, W, Dai, Y, Zheng, C, Liu, W, Yang, W, Shu, K. 2019.

Genome-wide identification of GRF transcription factors in soybean and expression analysis of GmGRF family under shade stress, BMC Plant Biology 19: 269.

Chou, KC, Shen, HB. 2010. Plant-mPLoc: A top-down strategy toaugment the power for predicting plant protein subcellular localization. PLoS ONE 5: e11335.

Conesa, A, Götz, S, García-Gómez, JM, Terol, J, Talón, M, Robles, M. 2005. Blast2GO: a universal tool for annotation, visualization and analysis in functional genomics research. Bioinformatics 21: 3674-3676.

Debernardi, JM, Mecchia, MA, Vercruyssen, L, Smaczniak, C, Kaufmann, K, Inze, D, Rodriguez, RE, Palatnik, JF. 2014. Post-transcriptional control of $G R F$ transcription factors by microRNA miR396 and GIF co-activator affects leaf size and longevity. The Plant Journal 79: 413-426.

El-Gebali, S, Mistry, J, Bateman, A, Eddy, SR, Luciani, A, Potter, SC, Qureshi, M, Richardson, LJ, Salazar, GA, Smart, A, Sonnhammer, EL L, Hirsh, L, Paladin, L, Piovesan, D, Tosatto, SC E, Finn, RD. 2018. The Pfam protein families database in 2019. Nucleic Acids Research 47: D427-D432.

Fang, ZW, He, YQ, Liu, YK, Jiang, WQ, Song, JH, Wang, SP, Ma, DF, Yin, JL. 2019. Bioinformatic identification and analyses of the non-specifc lipid transfer proteins in wheat. Journal of Integrative Agriculture 18: 2-17.

Fang, ZW, Jiang, WQ, He, YQ, Ma, DF, Liu, YK, Wang, SP, Zhang, YX, Yin, JL. 2020. Genomewide identification, structure characterization, and expression profiling of Dof transcription factor gene family in wheat (Triticum aestivum L.). Agronomy 10: 294.

Gao, F, Wang, K, Liu, Y, Chen, YP, Chen, P., Shi, ZY, Luo, J., Jiang, DQ, Fan, FF, Zhu, YG, Li, SP. 2015. Blocking miR396 increases rice yield by shaping inflorescence architecture. Nature Plants, 2: 15196.

He, LY, Liu, WY, Lou, YF, Xiao, FM. 2018. Genome-wide identification and analysis of the GRF 
467 468

469

470

471

472

473

474

475

476

477

478

479

480

transcription factor family in Moso bamboo (Phyllostachys edulis). Plant Science Journal 36: 713-720.

He, YQ, Huang, WD, Yang, L, Li, YT, Lu, C, Zhu, YX, Ma, DF, Yin, JL. 2020. Genome-wide analysis of ethylene-insensitive3 (EIN3/EIL) in Triticum aestivum. Crop science. DOI: $10.1002 / \csc 2.20115$

Heidel, AJ, Clarke, JD, Antonovics, J, Dong, X. 2004. Fitness costs of mutations affecting the systemic acquired resistance pathway in Arabidopsis thaliana. Genetics 168: 2197-2206.

Hewezi, T, Maier, TR, Nettleton, D, Baum, TJ. 2012. The Arabidopsis MicroRNA396$G R F 1 / G R F 3$ regulatory module acts as a developmental regulator in the reprogramming of root cells during cyst nematode infection. Plant Physiology 159: 321-335.

Horiguchi, G, Kim, GT., Tsukaya, H. 2005. The transcription factor AtGRF5 and the transcription coactivator AN3 regulate cell proliferation in leaf primordia of Arabidopsis thaliana. The Plant Journal 43: 68-78.

$\mathrm{Hu}, \mathrm{LF}$, Liu, SQ. 2011. Genome-wide identification and phylogenetic analysis of the ERF gene family in cucumbers. Genetics and Molecular Biology 34: 624-634.

Hu, LP, Zhang, F, Song, SH, Tang, XW, Xu, H, Liu, GM, Wang, Y, He, HJ. 2017. Genome-wide identification, characterization, and expression analysis of the SWEET gene family in cucumber. Journal of Integrative Agriculture 16: 1486-1501.

Inda, LA, Segarra-Moragues, JG, Müller, J, Peterson, PM, Catalán, P. 2008. Dated historical biogeography of the temperate Loliinae (Poaceae, Pooideae) grasses in the northern and southern hemispheres. Molecular Phylogenetics and Evolution 46: 932-957.

Jiang, WQ, Yang, L, He, YQ, Zhang, HT, Li, W, Chen, HG, Ma, DF, Yin, JL. 2019. Genomewide identification and transcriptional expression analysis of superoxide dismutase (SOD) family in wheat (Triticum aestivum). PeerJ 7: e8062.

Jiang, WQ, Geng, YP, Liu, YK, Chen, SH, Cao, SL, Li, W, Chen, HG, Ma, DF, Yin, JL. 2020. Genome-wide identification and characterization of SRO gene family in wheat: Molecular evolution and expression profiles during different stresses. Plant Physiology and Biochemistry 
154: 590-611.

Kim, JH, Choi, D, Kende, H. 2003. The AtGRF family of putative transcription factors is involved in leaf and cotyledon growth in Arabidopsis. The Plant Journal 36: 94-104.

Kim, JH, Kende, H. 2004. A transcriptional coactivator, AtGIF1, is involved in regulating leaf growth and morphology in Arabidopsis. Proceedings of the National Academy of Sciences of the United States of America 101: 13374-13379.

Kim, JH, Lee, BH. 2006. GROWTH-REGULATING FACTOR4 of Arabidopsis thaliana is required for development of leaves, cotyledons, and shoot apical meristem. Journal of Plant Biology 49: 463-468.

Kim, J-S, Mizoi, J, Kidokoro, S, Maruyama, K, Nakajima, J, Nakashima, K, Mitsuda, N, Takiguchi, Y, Ohme-Takagi, M, Kondou, Y, Yoshizumi, T, Matsui, M, Shinozaki, K, Yamaguchi-Shinozaki, K. 2012. Arabidopsis growth-regulating factor7 functions as a transcriptional repressor of abscisic acid- and osmotic stress-responsive genes, Including DREB2A. The Plant Cell 24: 3393-3405.

Kuijt, SJH, Greco, R, Agalou, A, Shao, J, 't Hoen, CCJ, Övernäs, E, Osnato, M, Curiale, S, Meynard, D, van Gulik, R, Maraschin, SdF, Atallah, M, de Kam, RJ, Lamers, GEM, Guiderdoni, E, Rossini, L, Meijer, AH, Ouwerkerk, PBF. 2014. Interaction between the growth-regulating factor and knotted1-like homeobox families of transcription Factors ${ }^{[\mathrm{W}]}$. Plant Physiology 164: 1952-1966.

Kumar, S, Stecher, G, Tamura, K. 2016. MEGA7: Molecular Evolutionary Genetics Analysis Version 7.0 for Bigger Datasets. Molecular Biology and Evolution 33: 1870-1874.

Lescot, M, Déhais, P, Thijs, G, Marchal, K, Moreau, Y, Van de Peer, Y, Rouzé, P, Rombauts, S. 2002. PlantCARE, a database of plant cis-acting regulatory elements and a portal to tools for in silico analysis of promoter sequences. Nucleic Acids Research 30: 325-327.

Letunic, I, Bork, P. 2017. 20 years of the SMART protein domain annotation resource. Nucleic Acids Research 46: D493-D496.

Letunic, I, Bork, P. 2019. Interactive Tree Of Life (iTOL) v4: recent updates and new 
521

522

523

524

525

526

527

528

529

530

531

532

533

534

535

536

537

538

539

540

541

542

543

544

545

546

547

developments. Nucleic Acids Research 47: W256-W259.

Li, R, An, JP, You, CX, Shu, J, Wang, XF, Hao, YJ. 2018. Identification and expression of the CEP gene family in apple (Malus $\times$ domestica). Journal of Integrative Agriculture 17: 348-358.

Ling, HQ, Zhao, SC, Liu, DC, Wang, JY, Sun, H., Zhang, C., Fan, HJ, Li, D., Dong, LL, Tao, Y., Gao, C., Wu, HL, Li, YW, Cui, Y., Guo, XS, Zheng, SS, Wang, B., Yu, K., Liang, QS, Yang, WL, Lou, XY, Chen, J., Feng, MJ, Jian, JB, Zhang, XF, Luo, GB, Jiang, Y., Liu, JJ, Wang, ZB, Sha, YH, Zhang, BR, Wu, HJ, Tang, DZ, Shen, QH, Xue, PY, Zou, SH, Wang, XJ, Liu, X., Wang, FM, Yang, YP, An, XL, Dong, ZY, Zhang, KP, Zhang, XQ, Luo, MC., Dvorak, J., Tong, YP, Wang, J., Yang, HM, Li, ZS, Wang, DW, Zhang, AM, Wang, J. 2013. Draft genome of the wheat A-genome progenitor Triticum urartu. Nature 496: 87-90.

Liu, HH, Tian, X, Li, YJ, Wu, CA, Zheng, CC. 2008. Microarray-based analysis of stress-regulated microRNAs in Arabidopsis thaliana. Rna 14: 836-843.

Liu, HH, Guo, SY, Xu, YY, Li, CH, Zhang, ZY, Zhang, DJ, Xu, SJ, Zhang, C, Chong, K. 2014. OsmiR396d-regulated OsGRFs function in floral organogenesis in rice through binding to their targets OsJMJ706 and OsCR4. Plant Physiology 165: 160-174.

Liu, J, Hua, W, Yang, HL, Zhan, GM, Li, RJ, Deng, LB, Wang, XF, Liu, GH, Wang, HZ. 2012. The BnGRF2 gene (GRF2-like gene from Brassica napus) enhances seed oil production through regulating cell number and plant photosynthesis. Journal of Experimental Botany 63: 37273740.

Liu, JY, Rice, JH, Chen, N, Baum, TJ, Hewezi, T. 2014. Synchronization of developmental processes and defense signaling by growth regulating transcription factors. PLoS ONE 9: e98477.

Liu, X, Guo, LX, Jin, LF, Liu, YZ, Liu, T, Fan, YH, Peng, SA. 2016. Identification and transcript profiles of citrus growth-regulating factor genes involved in the regulation of leaf and fruit development. Molecular Biology Reports 43: 1059-1067.

Ma, DF, Fang, ZW, Yin, JL, Chao, KX, Jing, JX, Li, Q, Wang, BT. 2016. Molecular mapping of stripe rust resistance gene $\mathrm{YrHu}$ derived from Psathyrostachys huashanica. Molecular Breeding 
36: 64.

549

550

551

552

553

554

555

556

557

558

559

560

561

562

563

564

565

566

567

568

569

570

571

572

573

574

Moore RC, Purugganan MD. 2003. The early stages of duplicate gene evolution. Proceedings of the National Academy of Sciences of the United States of America 100: 15682-15687.

Omidbakhshfard, MA, Proost, S, Fujikura, U, Mueller-Roeber, B. 2015. Growth-regulating factors (GRFs): A small transcription factor family with important functions in plant biology. Molecular Plant 8: 998-1010.

Ouyang, S, Zhu, W, Hamilton, J, Lin, H, Campbell, M, Childs, K, Thibaud-Nissen, F, Malek, RL, Lee, Y, Zheng, L, Orvis, J, Haas, B, Wortman, J, Buell, CR. 2006. The TIGR Rice Genome Annotation Resource: improvements and new features. Nucleic Acids Research 35: D883D887.

Paolacci, AR, Tanzarella, OA, Porceddu, E, Ciaffi, M. 2009. Identification and validation of reference genes for quantitative RT-PCR normalization in wheat. BMC Molecular Biology 10: 11.

Peng, JH, Sun, D, Nevo, E. 2011. Domestication evolution, genetics and genomics in wheat. Molecular Breeding 28: 281.

Ramírez-González, RH, Borrill, P, Lang, D, Harrington, SA, Brinton, J, Venturini, L, Davey, M, Jacobs, J, van Ex, F, Pasha, A, Khedikar, Y, Robinson, SJ, Cory, AT, Florio, T, Concia, L, Juery, C, Schoonbeek, H, Steuernagel, B, Xiang, D, Ridout, CJ, Chalhoub, B, Mayer, KFX, Benhamed, M, Latrasse, D, Bendahmane, A, Wulff, BBH, Appels, R, Tiwari, V, Datla, R, Choulet, F, Pozniak, CJ, Provart, NJ, Sharpe, AG, Paux, E, Spannagl, M, Bräutigam, A, Uauy, C. 2018. The transcriptional landscape of polyploid wheat. Science 361: eaar6089.

Sakuma, Y, Maruyama, K, Osakabe, Y, Qin, F, Seki, M, Shinozaki, K, Yamaguchi-Shinozaki, K. 2006. Functional analysis of an Arabidopsis transcription factor, DREB2A, involved in droughtresponsive gene expression. The Plant Cell 18: 1292-1309.

Schilling, S, Kennedy, A, Pan, S, Jermiin, LS, Melzer, R. 2020. Genome-wide analysis of MIKCtype MADS-box genes in wheat: pervasive duplications, functional conservation and putative neofunctionalization. New Phytologist 225: 511-529. 
575 Sun, C, Zhang, P, Fang, ZW, Zhang, X, Yin, JL, Ma, DF, Zhu, YX. 2019. Genetic analysis and 576 molecular mapping of stripe rust resistance in an excellent wheat line Sanshumai1. Journal of 577 Plant Pathology 101: 235-241.

578 Thompson, JD, Higgins, DG, Gibson, TJ. 1994. CLUSTAL W: improving the sensitivity of 579 progressive multiple sequence alignment through sequence weighting, position-specific gap 580 penalties and weight matrix choice. Nucleic Acids Research 22: 4673-4680.

581 van der Knaap, E, Kim, JH, Kende, H. 2000. A novel gibberellin-induced gene from rice and its 582 potential regulatory role in stem growth. Plant Physiology 122: 695-704.

583 Wang, FD, Qiu, NW, Ding, Q, Li, JJ, Zhang, YH, Li, HY, Gao, JW. 2014. Genome-wide 584 identification and analysis of the growth-regulating factor family in Chinese cabbage (Brassica 585 rapa L. ssp. pekinensis). BMC Genomics 15: 807.

586 Wu, L, Zhang, DF, Xue, M, Qian, JJ, He, Y, Wang, SC. 2014. Overexpression of the maize 587 GRF10, an endogenous truncated growth-regulating factor protein, leads to reduction in leaf 588 size and plant height. Journal of Integrative Plant Biology 56: 1053-1063.

589 Wang, YP, Tang, HB, DeBarry, JD, Tan, X, Li, JP, Wang, XY, Lee, T-h., Jin, HZ, Marler, B, Guo, 590 H, Kissinger, JC, Paterson, AH. 2012. MCScanX: a toolkit for detection and evolutionary 591 analysis of gene synteny and collinearity. Nucleic Acids Research 40: e49-e49.

592 Wu, ZJ, Wang, WL, Zhuang, J. 2017. Developmental processes and responses to hormonal stimuli 593 in tea plant (Camellia sinensis) leaves are controlled by $G R F$ and $G I F$ gene families. Functional 594 \& Integrative Genomics 17: 503-512.

595 Xu, GX, Guo, CC, Shan, HY, Kong, HZ. 2012. Divergence of duplicate genes in exon-intron 596 structure. Proceedings of the National Academy of Sciences 109: 1187-1192.

597 Yin, JL, Fang, ZW, Sun, C, Zhang, P, Zhang, X, Lu, C, Wang, SP, Ma, DF, Zhu, YX. $2018 \mathrm{a}$. 598 Rapid identification of a stripe rust resistant gene in a space-induced wheat mutant using specific 599 locus amplified fragment (SLAF) sequencing. Scientific Reports 8, 3086.

600 Yin, JL, Liu, M, Ma, D, Wu, J, Li, S, Zhu, Y, Han, B. 2018b. Identification of circular RNAs and 601 their targets during tomato fruit ripening. Postharvest Biology and Technology 136: 90-98. 
602 Yin, JL, Jia, JH, Lian, ZY, Hu, YH, Guo, J, Huo, HQ, Zhu, YX, Gong, HJ. 2019. Silicon enhances

603 the salt tolerance of cucumber through increasing polyamine accumulation and decreasing 604 oxidative damage. Ecotoxicology and Environmental Safety 169, 8-17.

605 Zhang, DF, Li, B, Jia, GQ, Zhang, TF, Dai, JR, Li, JS, Wang, SC. 2008. Isolation and 606 characterization of genes encoding GRF transcription factors and GIF transcriptional 607 coactivators in Maize (Zea mays L.). Plant Science 175: 809-817.

608 Zheng, XW, Yi, DX, Shao, LH, Li, C. 2017. In silico genome-wide identification, phylogeny and 609 expression analysis of the $R 2 R 3-M Y B$ gene family in Medicago truncatula. Journal of $610 \quad$ Integrative Agriculture 16: 1576-1591.

611 Zhu, YX, Xu, XB, Hu, YH, Han, WH, Yin, JL, Li, HL, Gong, HJ. 2015. Silicon improves salt 612 tolerance by increasing root water uptake in Cucumis sativus L. Plant Cell Reports 34: 16296131646.

614 Zhu, YX, Jia, JH, Yang, L, Xia, YC, Zhang, HL, Jia, JB, Zhou, R, Nie, PY, Yin, JL, Ma, DF, Liu, 615 LC. 2019a. Identification of cucumber circular RNAs responsive to salt stress. BMC Plant 616 Biology 19: 164.

617 Zhu, YX, Yang, L, Liu, N, Yang, J, Zhou, XK, Xia, YC, He, Y, He, YQ, Gong, HJ, Ma, DF, Yin, 618 JL. 2019b. Genome-wide identification, structure characterization, and expression pattern 619 profiling of aquaporin gene family in cucumber. BMC Plant Biology 19: 345.

620 Zhu, YX, Gong, HJ, Yin, JL. 2019c. Role of silicon in mediating salt tolerance in plants: a review. $621 \quad$ Plants 8: 147. 
Figure 1

Gene structure and motif analysis of TaGRF.

(A): The phylogenetic tree of TaGRF. This tree consists of 1000 bootstraps created by the Neighbor-Joining (NJ) method in MEGA7. (B): The motif of TaGRF was identified by MEME. MAST was used to display patterns. Each pattern is represented by a specific color. A red dot indicates a motif associated with a functional domain. (C): Exon-intron structure of TaGRF. Exon-intron structure analysis was performed using GSDS. The length of exons and introns is shown proportionally. Uuntranslated regions (UTR) are represented by blue boxes, exons are indicated by yellow boxes, and introns are indicated by black lines.

(A)

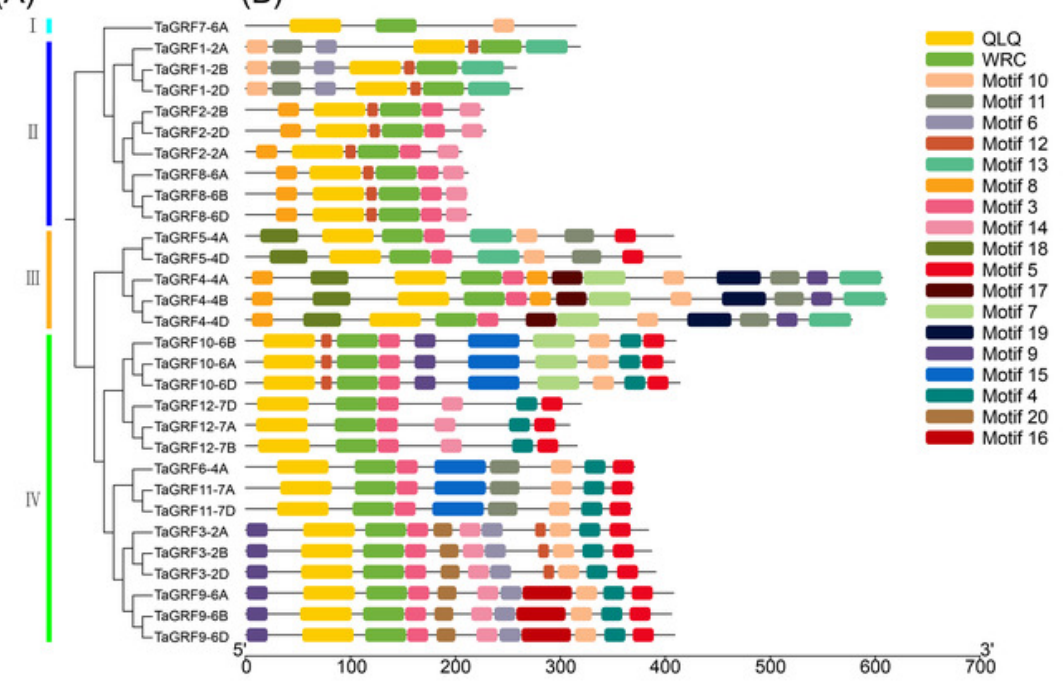

(C)

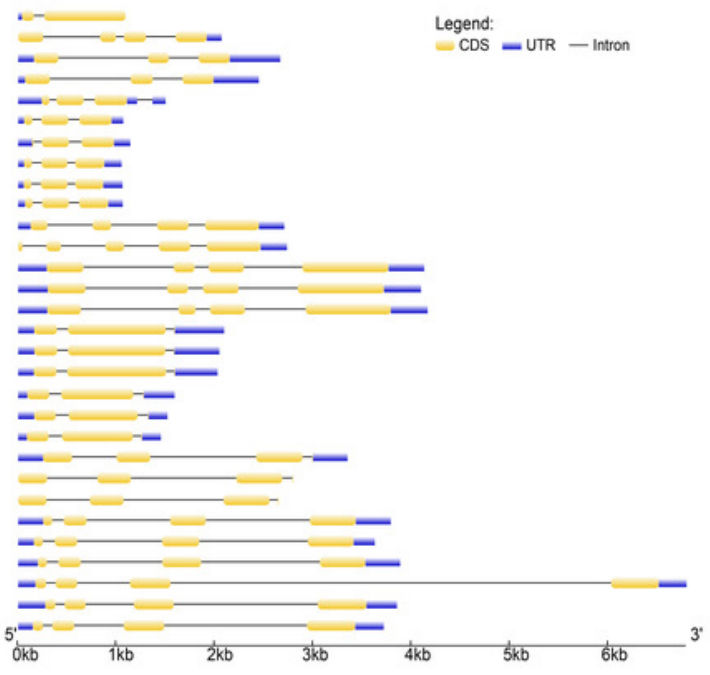


Figure 2

Protein sequence alignment of TaGRFs.

The functional areas are indicated by red boxes. 
TaGRE1-2A TaGRF1-2B TaGRF1-2D TaGRE2-2A TaGRE2-2B TaGRE2-2D TaGRE3-2A TaGRE3-2B TaGRF3-2D TaGRF $4-4 \mathrm{~A}$ TaGRE 4-4B TaGRF4-4D TaGRF 5-4A TaGRF5-4D TaGRE 6-4A TaGRE7-6A TaGRF 8-6A

TaGRE 8-6B TaGRF8-6D TaGRF9-6A

TaGRE9-6B TaGRE 9-6D TaGRF10-6 TaGRF10-6B TaGRF10-6D TaGRF11-7A TaGRF11-7D TaGRF 12-7A TaGRF12-7B TaGRF12-7D

TaGRF1-2A TaGRE1-2B TaGRE1-2D TaGRF2-2A TaGRE2-2B TaGRE 2-2D TaGRE3-2A TaGRF3-2B TaGRE3-2D TaGRF 4-4A TaGRE4-4B TaGRE4-4D TaGRE 5-4A TaGRF5-4D TaGRE 6-4A TaGRE7-6A TaGRF 8-6A TaGRF8-6B TaGRF8-6D TaGRF9-6A TaGRF9-6B TaGRE9-6D TaGRF10-6A TaGRF10-6B TaGRF10-6D TaGRF11-7A TaGRF11-7D TaGRF12-7A TaGRF12-7B TaGRF12-7D

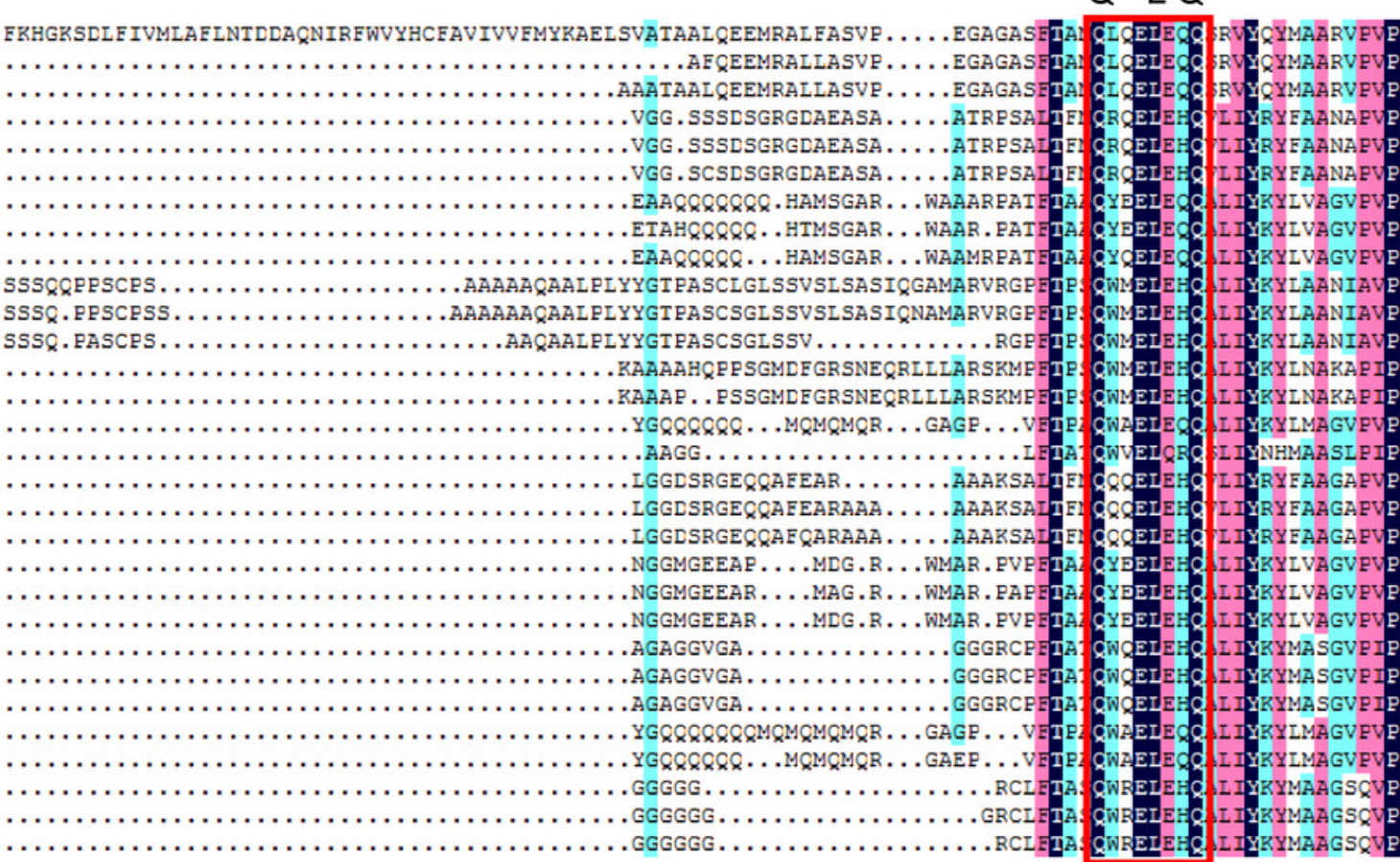

190

129

135

74

95

97

85

83

83

172

175

148

103

110

60

72

91

94

94

85

82

84

47

47

47

63

60

40

C WRC $\mathrm{CH}$

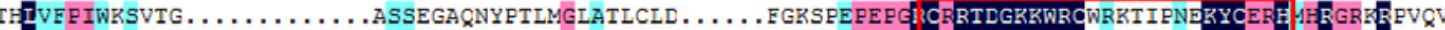

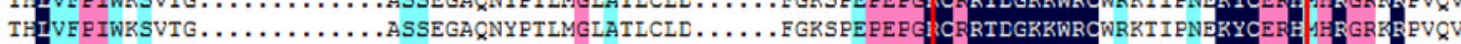

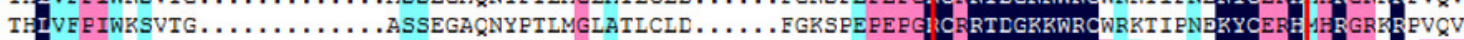

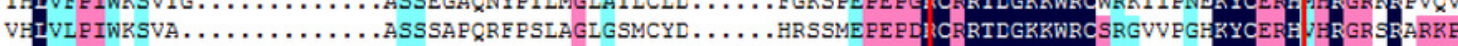

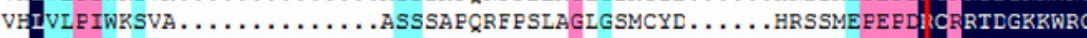

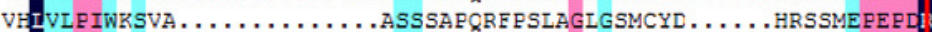

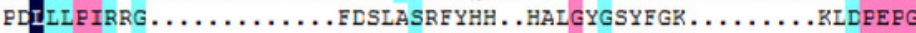

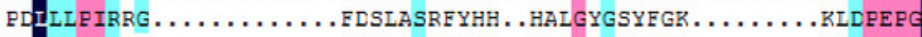

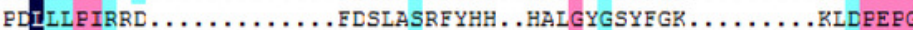
HSLLVPIRRSVT . . . . . . . . SLYRSAYFGSSTLGWGREQLG. . . YSGSADIERG

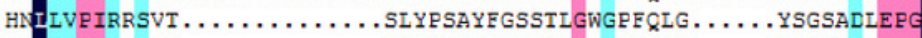

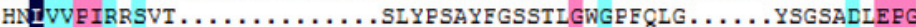
SGLIISISKSER $\ldots \ldots \ldots \ldots \ldots$ RSSDRMPWRPVYCGET $\ldots \ldots \ldots \ldots$ NADSDPEPG

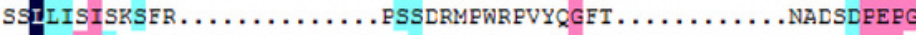
PDLLIPIRPHHPAAGAAGTTESEASPAASPEYHHHHPSMSYYAYYGR . . . . RLDPEP

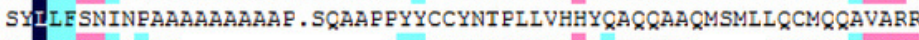
VHLVLPIWRSVA . . . . . . . . ASSEGPHRE PSLIGLGSLCFD . . . YRSSMEPEPG VHIVLPIWRSVA . . . . . . . . ASSEGPHREPSIIGLGSLCED . . . YRSSMEPEPG VNIVLPIWRSIA $\ldots \ldots \ldots \ldots \ldots$ ASSFGPHRE PSIIGLGSICED $\ldots \ldots$ YRSSMEPERG

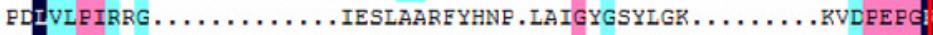

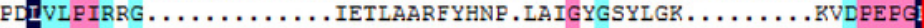

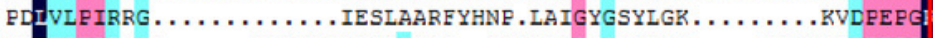

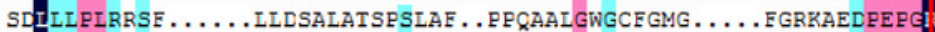

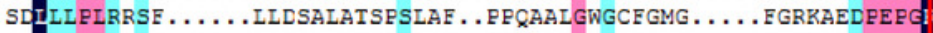

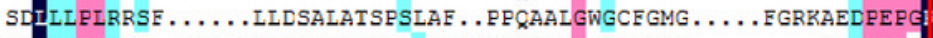
PDILLPIRPH . . PAG . AGATESEANPAASPFYHHHHPSMSYYAYYGR . . . . . RLDEEEK PDILIPIREH. . PAGAAGTTESEANPAASPFYHHHHPSMSYYAYYGK . . . . . RIDPERW

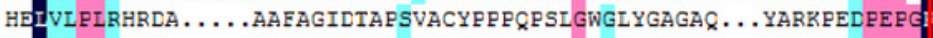
HEIVLPIRHRD . . . . . AAEAAIDTAPSLACYPREQRSIGWGLYGAGSQ . . YARKPEDPEPGRC RTDGKKWROSRGVVPGHYCED RTDGKKWRCSKEAACDSKYOERE THEGRNRSRKP RTDGKKWRC RTDGKKWRCSR RTDGKRWRCSRDAVAD QRYOERH 1 NRGRHRSRRH RTDGKRWRCSRDAVAD QRYCERH IN GRHR SRRH RTDGKKWRC SRDAVAD QRYOERH INRGRHRSRRH RTDGKKWRQSKEAMAEHRYCERH FNRNRHRSRRE RTLGKKWRCSKEAMAEFYYQERH FNRNRHRSRK RTDGKRWRCSKEAHPDSKYCERH HARGRNRSRRE

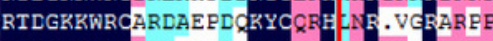
RTDGKKWRCSRDVVQGKKYGERH HHEGRGSRRE RTDGRKWRCSRDVVQGKRYQERH VHRGRGRSRKP RTDGKKWROSRDVLCQ CHRYOERE HHEGRGRSRRE RTLGKKWRCAREAASDSKYCERH 1 HR GRNRSRKR RTLGKRWROAREAASD SKYOERE 1HR GRNRSRKE RTDGKKWRCAKEAASDSKYCERH IHR GRN RSRRP RTDGKKWRCSKEAYPDSKYCEKH 1HRGKN SRKR RTDGKKWROSKEAYPDSKYCEKH 1HRGRNRSRKR RTDGKKWRCSKEAYPDSKYOEKH IHR GKN RSRKR RTDGKKWRCSKEAHPDSKYCERH 1HRGRN SRKR RTDGRKWRCSKEAHPDSKYCERH IHRGRNRSRRP RTDGKKWRCSREAYGESKYCDRH 1 HRGKN SRKR RTDGKKWRCSREAYGESKYCDRH 1 HRGRNR SRK

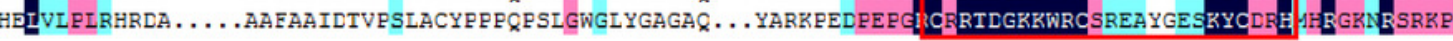


Figure 3

Chromosome locations of the 30 TaGRFs in wheat.

Different sub-groups of TaGRFs are represented in different colors: purple, Group I; blue, Group II; yellow, Group III; green, Group IV. In addition. Chr, Chromosome. The starting and ending information for the 30 chromosomal TaGRFs are listed in Annex 2. 

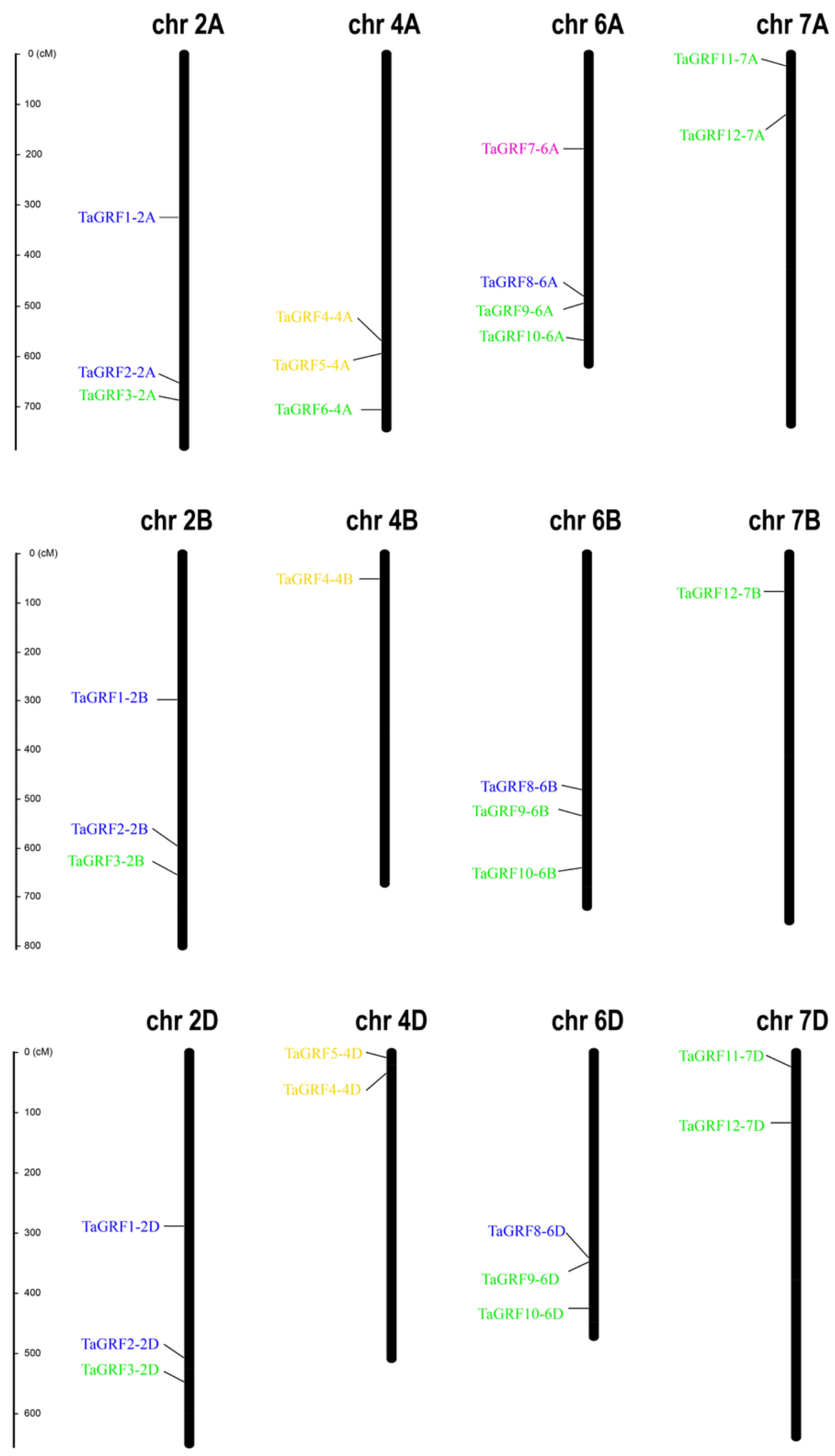


\section{Figure 4}

Phylogenetic tree of GRFs predicted in bread wheat and known in maize, rice, Arabidopsis, T. urartu, T. dicoccoides and Ae. tauschii.

All amino acid sequences were aligned using ClustalW. The phylogenetic tree was constructed by the Neighbor-Joining (1000 replicates) method using MEGA7.0. Different groups are distinguished by different colored ribbons. GRFs from wheat, maize, rice, Arabidopsis, T. urartu, T. dicoccoides and Ae. tauschii are distinguished with different colored circles.

\section{Colored ranges}

Arabidopsis thaliana

Oryza sativa

Triticum aestivum

Zea mays

Triticum urartu

Triticum dicoccum Aegilops tauschii

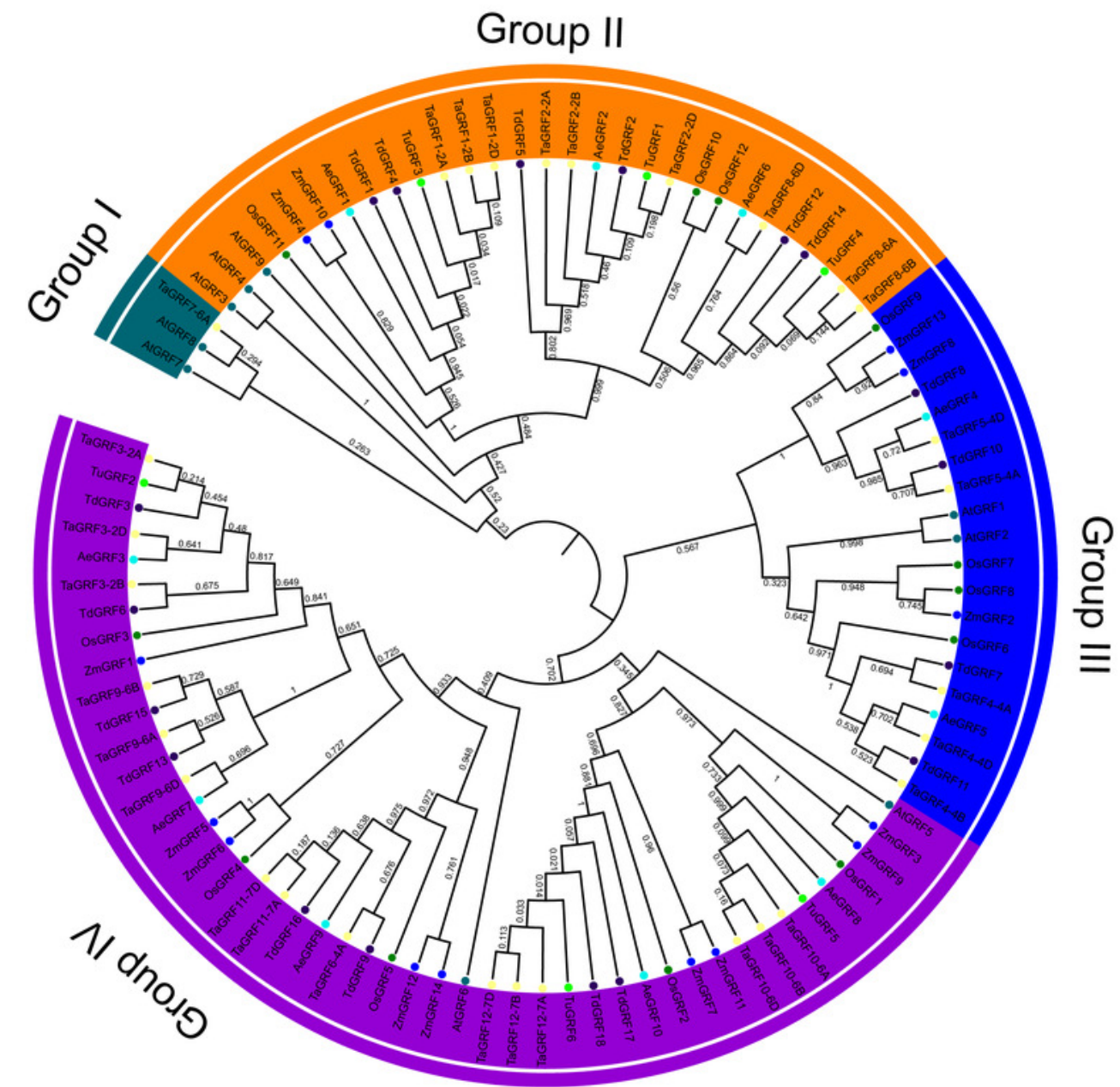


Figure 5

The cis-acting element involved in stress responses of the TaGRF genes promoters.

Different colors and numbers on the grid indicate numbers of different promoter elements in each TaGRF gene. All elements in the TaGRF gene promoter are listed in Annex 4.

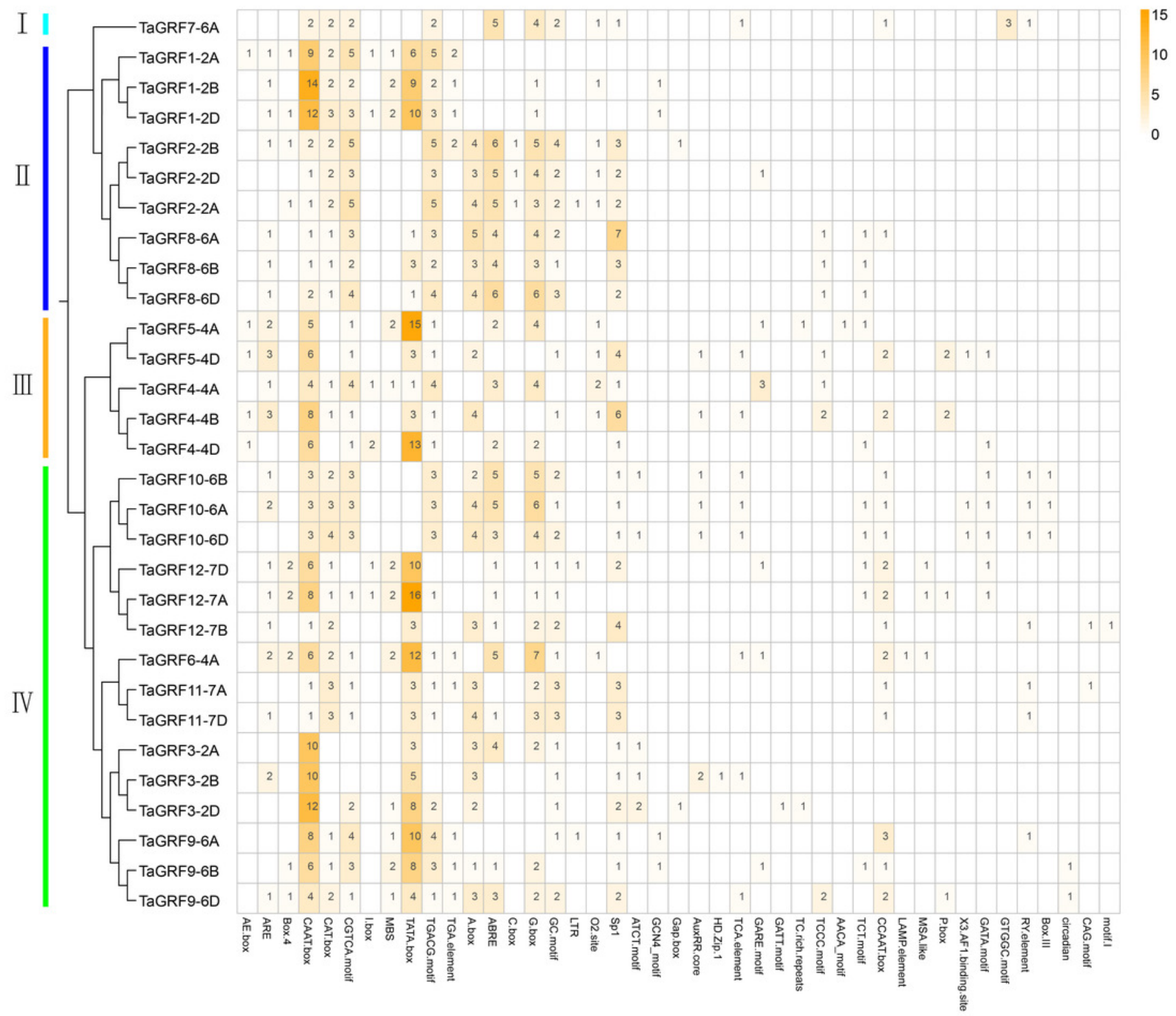


Figure 6

Gene Ontology (GO) distributions for the TaGRF proteins.

The gene ontology under three categories, biological processes, molecular functions and cellular component.

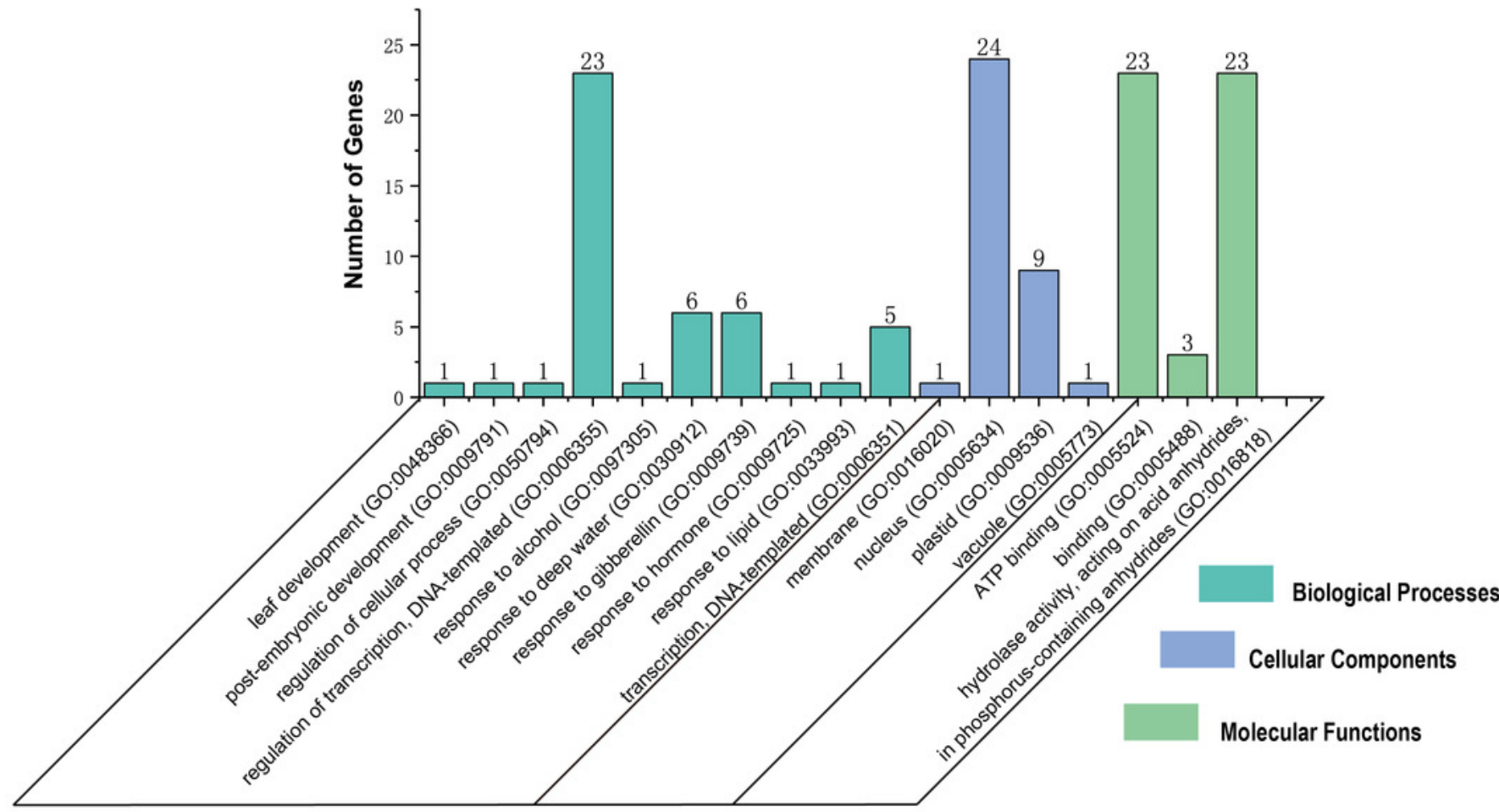

Biological Processes

Cellular Components Molecular Functions 


\section{Figure 7}

Synteny (A) and Phylogenetic (B) analyses for GRF genes in T. aestivum and its subgenomic progenitors $T$. urartu, $T$. dicoccoides and Ae. tauschii.

(A): Blue rectangles (Ae) represent Ae. tauschii chromosomes, Orange rectangles (Tu) represent $T$. urartu chromosomes, Yellow rectangles (Ta) represent $T$. aestivum chromosomes, Green rectangles (Td) represent $T$. dicoccoides chromosomes. (B): Phylogenetic relationship of $T$. aestivum, $T$. urartu, $T$. dicoccoides, and Ae. tauschii. This tree consists of 1000 bootstraps created by the Neighbor-Joining (NJ) method in MEGA7. The blue, yellow, green and purple circles represent $T$. dicoccoides, $T$. aestivum, Ae. tauschii, $T$. urartu, respectively. All identified GRF genes are in corresponding chromosomes (see Annex 2). The 89 homologous are shown in Annex 5.
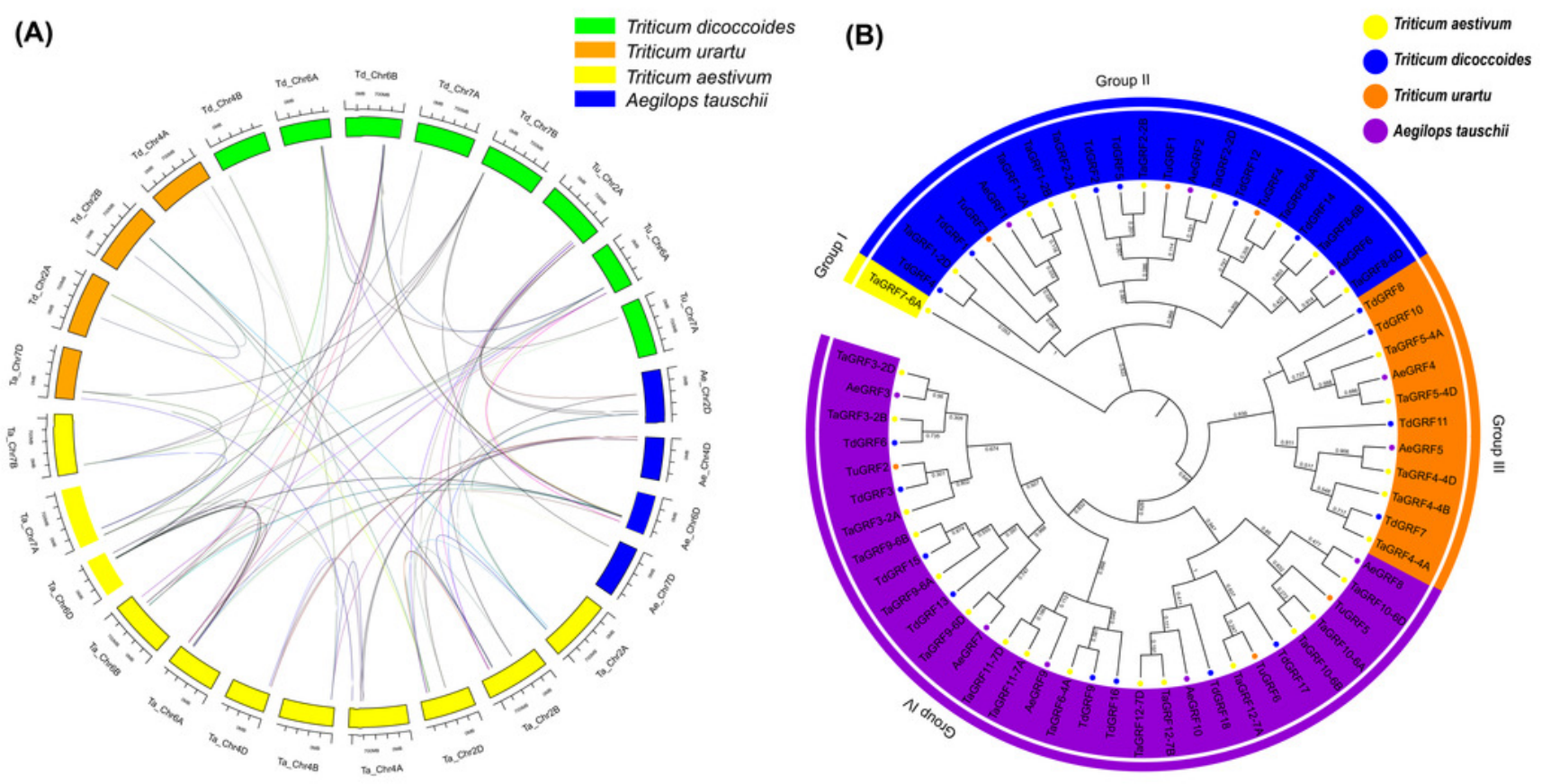
Figure 8

Transcriptome analyses of 30 TaGRFs.

(A): Growth and development. (B): Abiotic stresses.

(A)

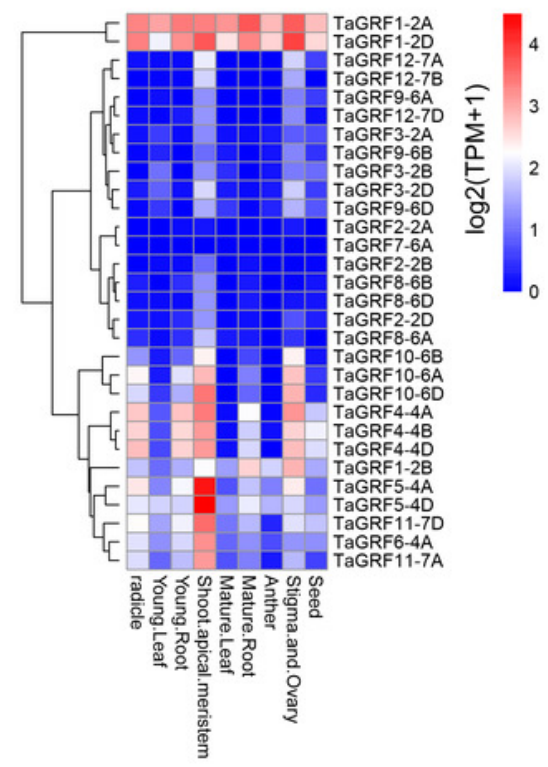

(B)

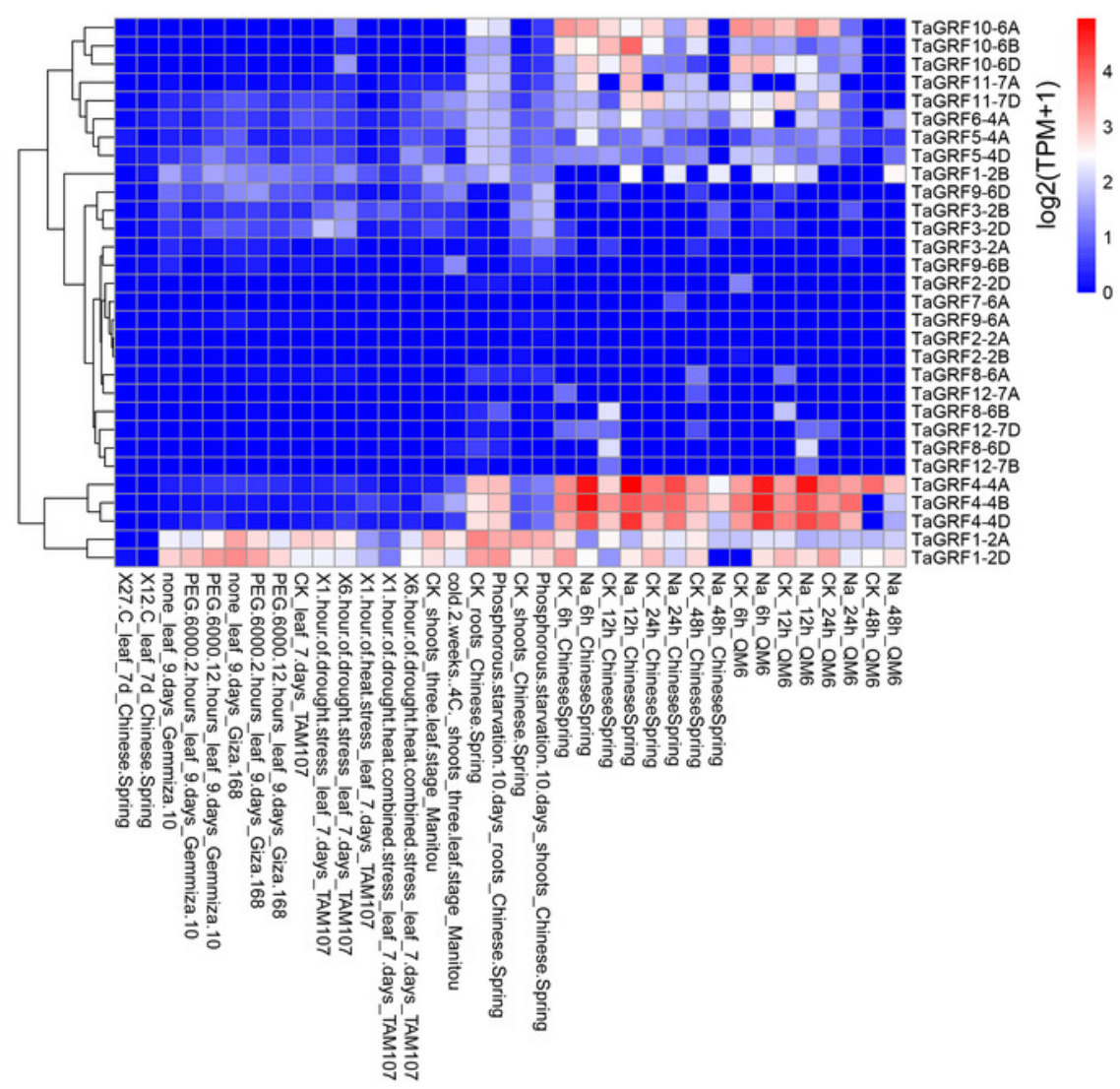


Figure 9

The qRT-PCR analyses of 14 TaGRFs in roots and leaves after treatment with $\mathrm{NaCl}$ and mannitol.

(A-N): NaCl root (O-BB): Mannitol root, (CC-PP): NaCl leaf, (QQ-DDD): Mannitol leaf. Time periods shown on the $\mathrm{x}$-axis. Expression levels are on the $\mathrm{y}$-axis. Standard deviations are shown with error bars. The expression levels of TaGRF genes were plotted using Origin software. 

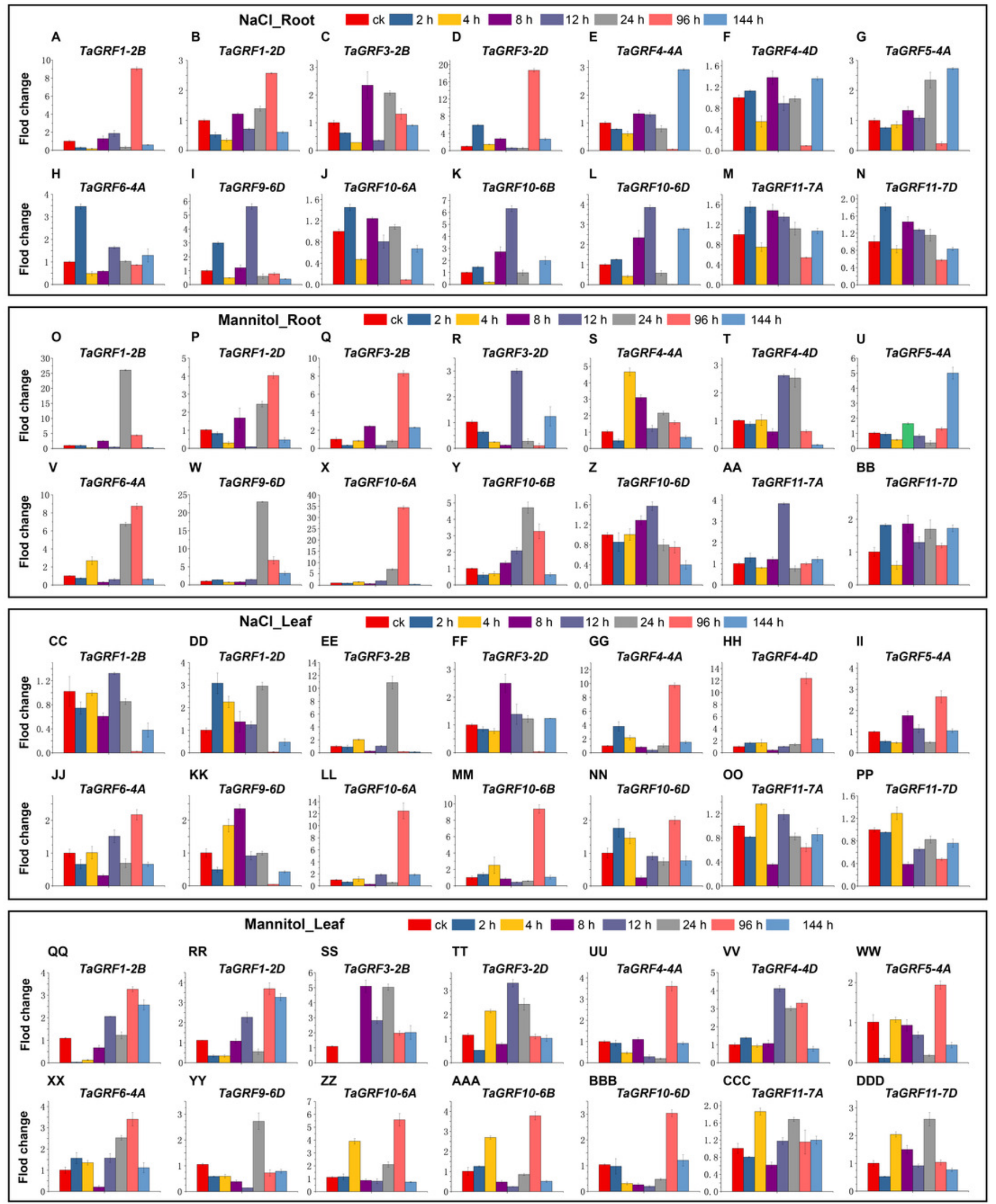
Table $\mathbf{1}$ (on next page)

Protein features of GRFs in Triticum aestivum 
Table 1. Protein features of GRFs in Triticum aestivum

\begin{tabular}{|c|c|c|c|c|c|c|c|c|}
\hline Name & Locus ID & $\begin{array}{l}\text { Le } \\
\text { n }\end{array}$ & MW & PI & II & stability & $\begin{array}{l}\text { GRAV } \\
\mathbf{Y}\end{array}$ & Sub \\
\hline TaGRF1-2A & TraesCS2A02G238700.1 & 319 & 34683.79 & 4.89 & 47.17 & unstable & -0.492 & $\begin{array}{l}\text { Chloroplast. Cytoplasm. } \\
\text { Nucleus. }\end{array}$ \\
\hline TaGRF1-2B & TraesCS2B02G256600.1 & 258 & 27746.68 & 4.72 & 54.92 & unstable & -0.736 & Nucleus. \\
\hline TaGRF1-2D & TraesCS2D02G246600.1 & 264 & 28182.2 & 4.76 & 55.93 & unstable & -0.692 & Nucleus. \\
\hline TaGRF2-2A & TraesCS2A02G398300.1 & 206 & 21620.58 & $\begin{array}{l}10.2 \\
3\end{array}$ & 49.79 & unstable & -0.269 & Chloroplast. Nucleus. \\
\hline TaGRF2-2B & TraesCS2B02G416300.1 & 227 & 24077.17 & 9.82 & 53.68 & unstable & -0.479 & Nucleus. \\
\hline TaGRF2-2D & TraesCS2D02G395900.1 & 229 & 24221.3 & 9.57 & 49.91 & unstable & -0.443 & Nucleus. \\
\hline TaGRF3-2A & TraesCS2A02G435100.1 & 384 & 42326.85 & 6.76 & 61.22 & unstable & -0.623 & Nucleus. \\
\hline TaGRF3-2B & TraesCS2B02G458400.1 & 387 & 42454.97 & 7.01 & 61.92 & unstable & -0.621 & Nucleus. \\
\hline TaGRF3-2D & TraesCS2D02G435200.1 & 391 & 42780.33 & 7.04 & 60.85 & unstable & -0.62 & Nucleus. \\
\hline TaGRF4-4A & TraesCS4A02G255000.1 & 607 & 63953.28 & 6.87 & 51.64 & unstable & -0.416 & Nucleus. \\
\hline TaGRF4-4B & TraesCS4B02G060000.1 & 611 & 64277.53 & 6.72 & 51.31 & unstable & -0.423 & Nucleus. \\
\hline TaGRF4-4D & TraesCS4D02G059600.1 & 578 & 61162.1 & 6.58 & 53.81 & unstable & -0.45 & Nucleus. \\
\hline TaGRF5-4A & TraesCS4A02G291500.1 & 408 & 45327.7 & 9 & 61.39 & unstable & -0.842 & Nucleus. \\
\hline TaGRF5-4D & TraesCS4D02G020300.1 & 415 & 45994.94 & 8.82 & 62.06 & unstable & -0.794 & Nucleus. \\
\hline TaGRF6-4A & TraesCS4A02G434900.1 & 371 & 39941.33 & 8.5 & 61.23 & unstable & -0.726 & Nucleus. \\
\hline TaGRF7-6A & TraesCS6A02G174800.1 & 315 & 33604.85 & 8.12 & 41.46 & unstable & -0.35 & Nucleus. \\
\hline TaGRF8-6A & TraesCS6A02G257600.1 & 212 & 22597.56 & 9.54 & 52.41 & unstable & -0.384 & Nucleus. \\
\hline TaGRF8-6B & TraesCS6B02G267500.1 & 211 & 22347.22 & 9.64 & 53.2 & unstable & -0.382 & Nucleus. \\
\hline TaGRF8-6D & TraesCS6D02G238900.1 & 215 & 22750.77 & 9.9 & 55.13 & unstable & -0.36 & Nucleus. \\
\hline TaGRF9-6A & TraesCS6A02G269600.1 & 408 & 43457.29 & 7.65 & 65.49 & unstable & -0.579 & Nucleus. \\
\hline TaGRF9-6B & TraesCS6B02G296900.1 & 406 & 43435.28 & 8.46 & 64.46 & unstable & -0.571 & Nucleus. \\
\hline TaGRF9-6D & TraesCS6D02G245300.1 & 409 & 43620.48 & 8.16 & 64.18 & unstable & -0.559 & Nucleus. \\
\hline $\begin{array}{l}\text { TaGRF10- } \\
6 \mathrm{~A}\end{array}$ & TraesCS6A02G335900.1 & 409 & 44786.45 & 7.22 & 51.42 & unstable & -0.833 & Nucleus. \\
\hline $\begin{array}{l}\text { TaGRF10- } \\
6 \mathrm{~B}\end{array}$ & TraesCS6B02G366700.1 & 410 & 44724.4 & 7.21 & 51.02 & unstable & -0.821 & Nucleus. \\
\hline $\begin{array}{l}\text { TaGRF10- } \\
6 \mathrm{D}\end{array}$ & TraesCS6D02G315700.1 & 414 & 45263.95 & 7.24 & 51.94 & unstable & -0.835 & Nucleus. \\
\hline $\begin{array}{l}\text { TaGRF11- } \\
7 \mathrm{~A}\end{array}$ & TraesCS7A02G049100.1 & 370 & 40161.6 & 8.78 & 59.57 & unstable & -0.762 & Nucleus. \\
\hline $\begin{array}{l}\text { TaGRF11- } \\
\text { 7D }\end{array}$ & TraesCS7D02G044200.1 & 368 & 39895.24 & 8.57 & 58.69 & unstable & -0.75 & Nucleus. \\
\hline $\begin{array}{l}\text { TaGRF12- } \\
\text { 7A }\end{array}$ & TraesCS7A02G165600.1 & 309 & 34214.05 & 8.55 & 65.62 & unstable & -0.841 & Nucleus. \\
\hline $\begin{array}{l}\text { TaGRF12- } \\
\text { 7B }\end{array}$ & TraesCS7B02G070200.1 & 316 & 34886.68 & 8.55 & 66.25 & unstable & -0.882 & Nucleus. \\
\hline
\end{tabular}


$\begin{array}{lllllllll}\text { TaGRF12- } & \text { TraesCS7D02G166400.1 } & 320 & 35405.24 & 8.26 & 63.68 & \text { unstable } & -0.882 & \text { Nucleus. } \\ \text { 7D } & \end{array}$

Len, Lengths (aa); MW, molecular weight (kD); pI, Isoelectric point; II, instability index; GRAVY, Grand average of hydropathicit; Sub, Subcellular localization.

1 